\title{
Analysis of Heterogeneous Cardiac Pacemaker Tissue Models and Traveling Wave Dynamics
}

\author{
Cheng Ly ${ }^{\mathrm{a}, *}$, Seth H. Weinberg ${ }^{\mathrm{b}, *}$ \\ ${ }^{a}$ Department of Statistical Sciences $\mathcal{E}$ Operations Research, Virginia Commonwealth University \\ ${ }^{b}$ Department of Biomedical Engineering, Virginia Commonwealth University
}

\begin{abstract}
The sinoatrial-node (SAN) is a complex heterogeneous tissue that generates a stable rhythm in healthy hearts, yet a general mechanistic explanation for when and how this tissue remains stable is lacking. Although computational and theoretical analyses could elucidate these phenomena, such methods have rarely been used in realistic (large-dimensional) gap-junction coupled heterogeneous pacemaker tissue models. In this study, we adapt a recent model of pacemaker cells (Severi et al., 2012), incorporating biophysical representations of ion channel and intracellular calcium dynamics, to capture physiological features of a heterogeneous population of pacemaker cells, in particular "center" and "peripheral" cells with distinct intrinsic frequencies and action potential morphology. Large-scale simulations of the SAN tissue, represented by a heterogeneous tissue structure of pacemaker cells, exhibit a rich repertoire of behaviors, including complete synchrony, traveling waves of activity originating from periphery to center, and transient traveling waves originating from the center. We use phase reduction methods that do not require fully simulating the large-scale model to capture these observations. Moreover, the phase reduced models accurately predict key properties of the tissue electrical dynamics, including wave frequencies when synchronization occurs, and wave propagation direction in a variety of tissue models. With the reduced phase models, we analyze the relationship between cell distributions and coupling strengths and the resulting transient dynamics. Further, the reduced phase model predicts parameter regimes of irregular electrical dynamics. Thus, we demonstrate that phase reduced oscillator models applied to realistic pacemaker tissue is a useful tool for investigating the spatial-temporal dynamics of cardiac pacemaker activity.
\end{abstract}

Keywords:

Sinoatrial-node, Traveling Waves, Heterogeneous pacemaker cells, Phase Reduction, Phase Oscillators

\section{Introduction}

The sinoatrial node (SAN) is the region of the heart responsible for generating the electrical rhythm. Although isolating individual cardiac pacemaker cells in the SAN to experimentally

\footnotetext{
${ }^{*}$ Corresponding authors

Email addresses: cly@vcu . edu (Cheng Ly), shweinberg@vcu . edu (Seth H. Weinberg)

URL: www. shweinberglab.com (Seth H. Weinberg)
}

Preprint submitted to Journal of Theoretical Biology 
measure electrophysiological properties is feasible (Honjo et al., 1996, Kodama et al., 1997), a detailed understanding of the dynamics of intact coupled networks of cardiac cells (i.e., tissue) is difficult for several reasons. The SAN structure is highly complex at both the cellular and tissue levels, with heterogeneous cell populations and gap junctional coupling. During normal sinus rhythm, spontaneous electrical activity, driven by the pacemaker cells, propagates throughout the SAN tissue, typically in a center-to-periphery spatial pattern, and then activating the surrounding atrial tissue. While previous experimental studies have investigated SAN function (Fedorov et al., 2009, Efimov et al., 2010, Fedorov et al., 2010, 2012, Glukhov et al., 2013, Li et al., 2015, Csepe et al., 2016, Li et al., 2017), the mechanisms of how regular rhythms are generated in this complex tissue structure are still not well understood. Computational modeling can play a valuable role in understanding the dynamics of healthy and diseased cardiac rhythms (GreerShort et al., 2017, Phadumdeo and Weinberg, 2018; Arevalo et al., 2016, Oren and Clancy, 2010; Zhang et al., 2000, Wilders, 2007; Joyner and Van Capelle, 1986), especially as models increase in complexity to incorporate new biological details (Severi et al., 2012).

There is some uncertainty as to the degree of cellular heterogeneity within the cell population comprising the SAN tissue. Structural studies have described a "central" SAN region of smaller cells, with a transition to larger "peripheral" SAN cells (Bouman and Jongsma, 1986), with distinct action potential and calcium handling properties distinguishing these cell types (Honjo et al. 1996, Kodama et al. 1997). However, other studies have not found distinct isolated SAN cell electrical and calcium properties based on cell size (Michaels et al., 1987, Verheijck et al., 1998). In this study, we will investigate electrical properties in the SAN tissue, considering these two different approaches: SAN tissue comprised of (1) a homogeneous population of either all "center" cells or all "peripheral" cells (described further below); or (2) a heterogeneous population that transitions from center to peripheral cells.

While realistic large-scale SAN tissue models can yield insightful simulations, these systems are typically too complicated for detailed mechanistic descriptions of phenomena; a mathematical framework could aid this endeavor. To our knowledge, all computational studies of SAN tissue (Oren and Clancy, 2010, Severi et al., 2012, Zhang et al., 2000, Wilders, 2007; Inada et al. 2014) rely on tissue-scale simulations, without mechanistic mathematical analysis describing the critical properties that regulate cardiac electrical rhythms. There are mathematical studies of simplified SAN models, but without direct correspondence to large-scale biophysical models (see Discussion).

In this study, we apply mathematical methods for studying coupled heterogeneous oscillators (pacemaker cells) to analyze a novel minimal model of SAN tissue electrical activity. Our coupled oscillator model is developed from the periodic dynamics of a recent multi-compartment biophysical pacemaker model from Severi et al. (2012), with realistic calcium dynamics and ionic currents. The Severi et al. model was formulated to address limitations of previous pacemaker cell models, specifically reproducing the effects of perturbations to both ionic currents and calcium handling. Following prior work (Zhang et al., 2000, Kurata et al., 2002; Oren and Clancy, 2010), cellular heterogeneity attributes (i.e., differences between center and peripheral cells) in uncoupled cardiac pacemaker cells are represented by varying model cell size and ionic conductances. We then apply the theory of weakly coupled phase oscillators (Kopell and Ermentrout, 1986, Ermentrout, 1992) to formulate a reduced phase model with a 1D of "chain" of coupled phase oscillators, minimally representing the fully coupled SAN tissue in a manner that does not require simulating the full tissue model.

We compare this reduced model with a biophysically detailed SAN tissue model with hundreds of cells, coupled via gap junctions. Computationally expensive simulations of several 
coupled SAN tissue models varying from homogeneous to heterogeneous cell populations results in a variety of spatio-temporal dynamics, including: persistent synchrony, persistent traveling waves that originate in the periphery, transient traveling waves the originate in the center, etc. Our formulation of a 1D chain of phase oscillators captures the persistent wave frequency, synchronization, and wave propagation direction in a variety of large-scale SAN tissue models. Furthermore, this mathematical framework can predict the existence and stability of traveling waves and characterize the duration of transient activity before settling to a persistent state. We demonstrate that this phase reduction method can qualitatively and, in some cases, quantitatively reproduce electrical properties of biophysically realistic and heterogeneous coupled SAN tissue.

\section{Methods}

\section{Detailed Biophysical Pacemaker Cell Models}

The SAN isolated cell and tissue models are based on the recent rabbit SAN model from Severi et al. (2012), with the membrane potential for cell $j\left(v_{j}\right)$ governed by the following currentbalance equation (Oren and Clancy, 2010; Inada et al., 2014),

$$
C_{j} \frac{d v_{j}}{d t}=-I_{N a}-I_{f}-I_{t o}-I_{C a, L}-I_{C a, T}-I_{K r}-I_{K s}-I_{N a, K}-I_{N a, C a}-g_{g a p} \sum_{k}\left(v_{j}-v_{k}\right),
$$

where $C_{j}$ is membrane capacitance and which incorporates biophysical descriptions of ionic currents (see Table 1 for names of currents), a detailed model of calcium handling including sarcoplasmic reticulum (network and junctional), and ionic current pumps and exchangers. There are 31 state variables for each pacemaker cell; see Appendix A for further details. The last term represents local coupling via gap junctions, $g_{g a p} \sum_{k}^{1}\left(v_{j}-v_{k}\right)$, between cell $j$ and cell $k$. Gap junctional conductance $g_{g a p}$ is only non-zero if cells $j$ and $k$ are coupled and further depend on distance from the tissue center in the heterogeneous coupling schemes considered. Note that Eq. (1) assumes that cells are isopotential and that cytoplasmic resistance is neglected since it is much smaller than the gap junctional resistance.

For center cells, we use the same parameters as Severi et al. (2012) to match the reported action potential characteristics described in Zhang et al. (2000); Kodama et al. (1997); Oren and Clancy (2010). For the other types of pacemaker cells (i.e., transitioning from center to peripheral cells, see Fig. 11), we vary the following 12 parameters: cell capacitance, cell length, cell radius, and the maximum conductance of the funny currents, L-type and T-type calcium currents, slow and rapid delayed rectifier potassium currents, sodium current, sodium-potassium pump, and sodium-calcium exchanger. Parameter values for center and peripheral cells (Table 2) are based on values reported in Oren and Clancy (2010), and scale factors in Zhang et al. (2000).

A model of the SAN tissue is represented by 271 Severi pacemaker cells with coupling via nearest neighbor gap junctions (see Fig. $2 \mathrm{C}$ left). The SAN tissue is represented by a $2 \mathrm{D}$ hexagonal grid of uniformly spaced cells, i.e., mimicking a radially symmetric architecture, while maintaining the same Euclidean distance between all neighboring cells, to more closely agree with the lower dimensional reduced phase model. Simulation of the full biophysical representation of the heterogeneous SAN tissue are computationally expensive, requiring several days to simulate $20 \mathrm{~s}$ of biological time for a single parameter set. The required computational resources (on a High Performance Computing cluster with 64-bit AMD computer cores and clock 
speeds ranging between 2.1 to $2.6 \mathrm{GHz}$ ) are large because the MATLAB (The Mathworks, Natick, MA) stiff ODE solver (ode15s) was used with very small Relative and Absolute Tolerances of $1 \times 10^{-6}$; simulations of $20 \mathrm{~s}$ of biological time were used to resolve the periodic state with high accuracy. Note that histology within the SAN is highly irregular and in our model, cell size varies throughout the hexagonal cell arrangement; however we can estimate the SAN tissue area as $\sim 1.5 \mathrm{~mm}^{2}$ (corresponding to a hexagon with a diameter of 19 cells or $\sim 1.5 \mathrm{~mm}$ ), which is smaller than the typical rabbit SAN tissue area of $\sim 2 \mathrm{~mm}$ x $5 \mathrm{~mm}$, or $10 \mathrm{~mm}^{2}$ (Östborn, 2002).

Table 1: Definition of currents in Eq (1).

\begin{tabular}{cl}
\hline Equation & Name \\
\hline \hline$I_{N a}$ & Sodium current \\
\hline$I_{f}$ & Funny current \\
\hline$I_{t o}$ & Transient outward potassium current \\
\hline$I_{C a, L}$ & L-type calcium current \\
\hline$I_{C a, T}$ & T-type calcium current \\
\hline$I_{K r}$ & Rapid delayed rectifier $\mathrm{K}^{+}$current \\
\hline$I_{K s}$ & Slow delayed rectifier $\mathrm{K}^{+}$current \\
\hline$I_{N a, K}$ & Sodium-potassium pump \\
\hline$I_{N a, C a}$ & Sodium-calcium exchanger \\
\hline
\end{tabular}

\section{Phase Reduced Model}

To mathematically analyze the persistent (or steady-state traveling wave) behavior of the coupled pacemaker cells, we employ phase reduction methods, in which the dynamics of high dimensional systems are approximated with a simple scalar periodic variable. These methods have been successfully applied to many areas of science (Winfree, 1967), including chemical oscillations (Kuramoto, 2012), synchronization of fireflies (Mirollo and Strogatz, 1990), circadian rhythms (Winfree, 1970, Zeitzer et al., 2000), and cellular networks (Ermentrout and Terman 2010, Schultheiss et al. 2011; Ly and Ermentrout, 2010), to name a few. We briefly describe the general approach here (see Chapter 8 of Ermentrout and Terman (2010)). Consider a large dimensional model $X_{j} \in \mathbb{R}^{n}$ coupled to $X_{k} \in \mathbb{R}^{n}$ of the form:

$$
\frac{d X_{j}}{d t}=F\left(X_{j}\right)+\varepsilon G\left(X_{j}, X_{k}\right)
$$

When there is an asymptotically stable limit cycle $X_{0}(t)$ with period $T_{j}$, for the uncoupled case $\varepsilon=0$, we map values near the limit cycle to the unit circle via a function $\phi\left(X_{j}\right):=\Theta_{j} \in\left[0, T_{j}\right)$, to derive the following:

$$
\frac{d \Theta_{j}}{d t}=1+\nabla_{X} \phi\left(X_{j}\right) \cdot \varepsilon G\left(X_{j}, X_{k}\right)
$$

Since other cells $X_{k}$ only directly effect the voltage variable, all components of $\nabla_{X} \phi\left(X_{j}\right) \cdot \varepsilon G\left(X_{j}, X_{k}\right)$ are 0 except the voltage component. For small $\varepsilon$, we approximate $\nabla_{X} \phi\left(X_{j}\left(\Theta_{j}\right)\right) \approx \nabla_{X} \phi\left(X_{0}\left(\Theta_{j}\right)\right)$; the voltage component of this is the infinitesimal phase-resetting curve (PRC), which we denote by $\Delta_{j}\left(\Theta_{j}\right)$. Next, the method of averaging is applied to approximate the second term of Eq (2) with the function:

$$
H_{j, k}\left(\Theta_{k}-\Theta_{j}\right):=\frac{1}{T_{j}} \int_{0}^{T_{j}} \Delta_{j}(t) G\left(X_{j}(t), X_{k}\left(t+\Theta_{k}-\Theta_{j}\right)\right) d t,
$$


often called the interaction function (or $\mathrm{H}$-function). Note that PRC of cells can be experimentally measured by injecting pulses at various phases and recording the times to next spike (see Sano et al. (1978); Jalife et al. (1980) who measured PRCs in SAN pacemaker cells of mammals); the infinitesimal PRC is the limit of small (i.e., zero) duration and amplitude pulses.

This phase reduction can be applied repeatedly for each of the different pacemakers, with a rescaling of time so that $\theta_{j} \in[0,1)$ to arrive at the following phase network approximation:

$$
\frac{d \theta_{j}}{d t}=\omega_{j}+\sum_{k} H_{j, k}\left(\theta_{k}-\theta_{j}\right)
$$

Since we are interested in the direction of the traveling wave (if it exists) and the large SAN model is nearly radially symmetric in cell type, the phase model is further simplified to a 1-dimensional chain with "cut-ends", with one end being the Center and the other being the Peripheral (see Fig. 3C). Furthermore, a relatively large number of cell types (9) are used to span the range from a prototypical center pacemaker to peripheral pacemaker, enabling us to assume a modest amount of heterogeneity among neighboring cells (see Fig. 1B). Since coupling is local via gap junctions, we approximate the interaction function (for $k=j-1$ and $k=j+1$ only):

$$
H_{j, k}(x) \approx H_{j}(x)
$$

where $H_{j}$ is the interaction function of the $j^{\text {th }}$ cell type with itself, which can be numerically computed with the program XPP (Ermentrout, 2002). Specifically, we first compute the infinitesimal PRC $\Delta_{j}(t)$ in Eq. (4) via XPP (the mechanics of which are described in Chapter 9.5.1 of Ermentrout (2002)), after which the H-function is computed with an automated subroutine in XPP (described in Chapter 9.5.2 of Ermentrout (2002)). Note that the required XPP file is publicly available at http://github.com/chengly70/SanHeteroSeveri

In order to systematically relate the 1D chain of phase oscillators to the large scale model, each of the cell types are grouped together, and an average conductance is calculated via:

$$
\bar{g}_{g a p, j}=\frac{1}{\# \text { nonzero } g_{j, k}} \sum_{k} g_{j, k} .
$$

Here, $g_{j, k}$ denotes the $g_{g a p}$ conductance (see Eq. (1)) between cell $j$ and cell $k$ (Fig. 2 $\mathrm{C}$ left color-codes all 9 cell types by spatial location). This value of $\bar{g}_{g a p, j}$ multiplies the corresponding pieces of the interaction functions (plotted in Fig. $3 \mathbf{B}$ ):

$$
H_{j, k} \approx \bar{g}_{g a p, j} H_{j}
$$

We can use this phase reduction approach to determine the existence of traveling wave solutions. These solutions to equation (5) are of the form:

$$
\theta_{j}(t)=\Omega t+\xi_{j}
$$

\section{Quantifying Transient Times with Phase Models}

If a stable persistent state exists for a given phase reduction and tissue structure, we are also interested in the time required for the system to reach this persistent state, following a perturbation or from a given initial condition. We can calculate this transient time in the full 
SAN tissue model via simulation and compare with the reduced phase model framework. In the reduced phase model, consider a perturbation $\vec{\eta}(t)$ to a traveling wave solution $\Omega t+\vec{\xi}$

$$
\theta_{j}(t)=\Omega t+\xi_{j}+\eta_{j}(t)
$$

After substituting the above equation into Eq. $(5)$ and assuming perturbations are small $\left(\eta_{j}=\right.$ $O(\varepsilon)$ for all $j$, different $\varepsilon$ than in Eq. (2)), the $1^{s t}$ order $\varepsilon$ equation provides a linear approximation for the dynamics of $\eta_{j}(t)$ :

$$
\begin{aligned}
\frac{d \eta_{1}}{d t} & =H_{1,2}^{\prime}\left(\xi_{2}-\xi_{1}\right)\left(\eta_{2}-\eta_{1}\right) \\
\frac{d \eta_{j}}{d t} & =H_{j, j-1}^{\prime}\left(\xi_{j-1}-\xi_{j}\right)\left(\eta_{j-1}-\eta_{j}\right)+H_{j, j+1}^{\prime}\left(\xi_{j+1}-\xi_{j}\right)\left(\eta_{j+1}-\eta_{j}\right) ; \text { for } j=2, \ldots, 8 \\
\frac{d \eta_{9}}{d t} & =H_{9,8}^{\prime}\left(\xi_{8}-\xi_{9}\right)\left(\eta_{8}-\eta_{9}\right)
\end{aligned}
$$

This equation in matrix-vector form is:

$$
A:=\left(\begin{array}{ccccccc}
-H_{1,2}^{\prime} & H_{1,2}^{\prime} & 0 & \ldots & \ldots & \ldots & 0 \\
\vdots & \vdots & \vdots & \vdots & \vdots & \vdots & \\
0 & \cdots 0 & H_{j, j-1}^{\prime} & -H_{j, j-1}^{\prime}-H_{j, j+1}^{\prime} & H_{j, j+1}^{\prime} & 0 \cdots & 0 \\
\vdots & \vdots & \vdots & \vdots & \vdots & \vdots & \\
0 & \ldots & \ldots & \ldots & 0 & H_{9,8}^{\prime} & -H_{9,8}^{\prime}
\end{array}\right)
$$

Each of the terms in the matrix abbreviated as: $H_{j, k}^{\prime}:=H_{j, k}^{\prime}\left(\xi_{k}-\xi_{j}\right)$. When a traveling wave solution is stable, all but one of the eigenvalues of $A$ have negative real part; there is a distinct zero eigenvalue $\lambda_{0}=0$ with eigenvector $\overrightarrow{1}$ that corresponds to a constant shift in the traveling wave solution 1

Let the eigenvectors of $A$ be $\vec{\psi}_{j}$ with eigenvalues $\lambda_{j}: A \vec{\psi}_{j}=\lambda_{j} \vec{\psi}_{j}$, then since $A$ is symmetric, we take $\vec{\psi}_{j}$ to be an orthonormal set. With initial condition $\vec{\eta}_{0}$, the vector of perturbations is captured using an eigenvector expansion to solve for:

$$
\frac{d \vec{\eta}}{d t}=A \vec{\eta}
$$

to get:

$$
\vec{\eta}(t)=\sum_{k \neq 0}\left(\vec{\psi}_{k}, \vec{\eta}_{0}\right) e^{\lambda_{k} t} \vec{\psi}_{k}
$$

The solution can be approximated by using only the largest real eigenvalue with an eigenvector expansion (excluding the 0 eigenvector/value):

$$
\vec{\eta}(t)=\left(\vec{\psi}_{1}, \overrightarrow{\eta_{0}}\right) e^{\lambda_{1} t} \vec{\psi}_{1}
$$

The operation $(\vec{v}, \vec{w})$ is the usual inner product. Fig S2 (S1Text.pdf) shows that this approximation is excellent for the tissues and initial condition we consider.

\footnotetext{
${ }^{1} \mathrm{~A}$ constant shift in all variables is a perturbation that does not decay, because the result is the same traveling wave solution.
} 


\section{Results}

\subsection{Large-scale Pacemaker Tissue}

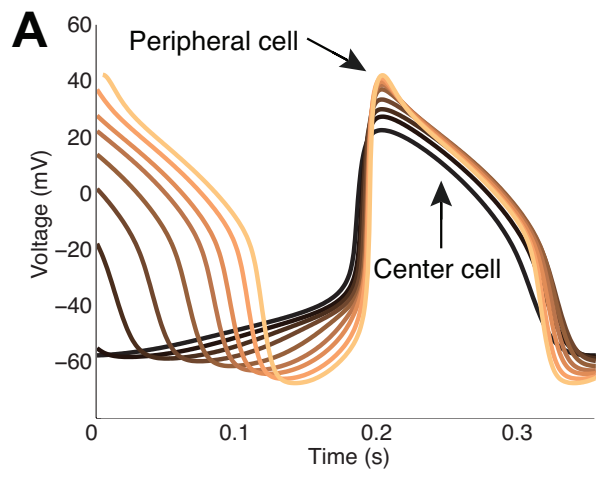

B

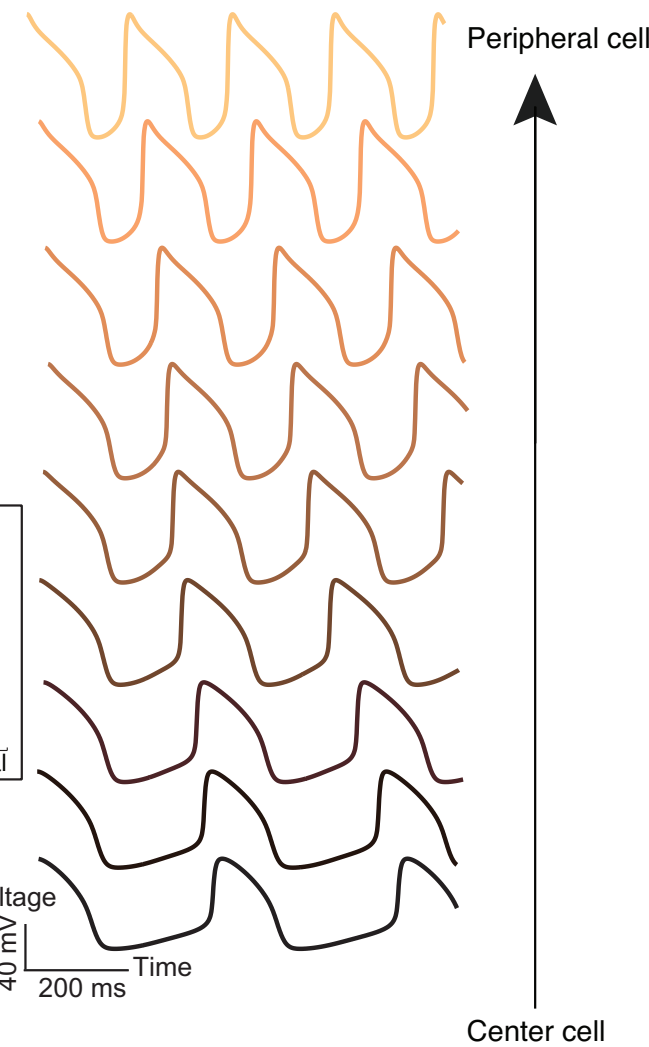

Figure 1: The full cardiac pacemaker model, showing all 9 heterogeneous cell types (uncoupled). A) The spike wave form for all 9 cells, shifted so the peaks are at the same time point to contrast waveforms. B) Voltage trace of the model over several beats for all 9 cell types. C) The cycle length (in seconds); the inset shows the frequency in 1/s.

We first developed a population of physiologically realistic pacemaker cell models, using the Severi et al. (2012) model as the baseline center cell. This model includes a variety of gating variables, ionic currents, and cellular voltage and calcium dynamics. By altering 12 parameters, our modified model reproduces the wide-variety of intrinsic frequencies and action potential morphology of different pacemaker cell types. While there is still much debate about the degree of heterogeneity within the SAN cell population, prior experimental work has shown that larger peripheral cells have faster intrinsic frequencies than smaller center cells, as well as other electrophysiological properties (Honjo et al., 1996, Kodama et al., 1997, Opthof et al., 1987). The uncoupled frequency of peripheral cells is almost double $(\approx 1.8 \times$ larger than $)$ the center cells (Fig. 11 C inset), so the weak heterogeneity assumption (Eq.66) does not strictly hold. Additionally, action potential morphology is altered, as the voltage trace has a smaller minimum and larger maximum and overall shorter action potential duration in peripheral cells, compared 
with center cells (Fig. 1A), consistent with experimental recordings (Honjo et al., 1996; Kodama et al., 1997).
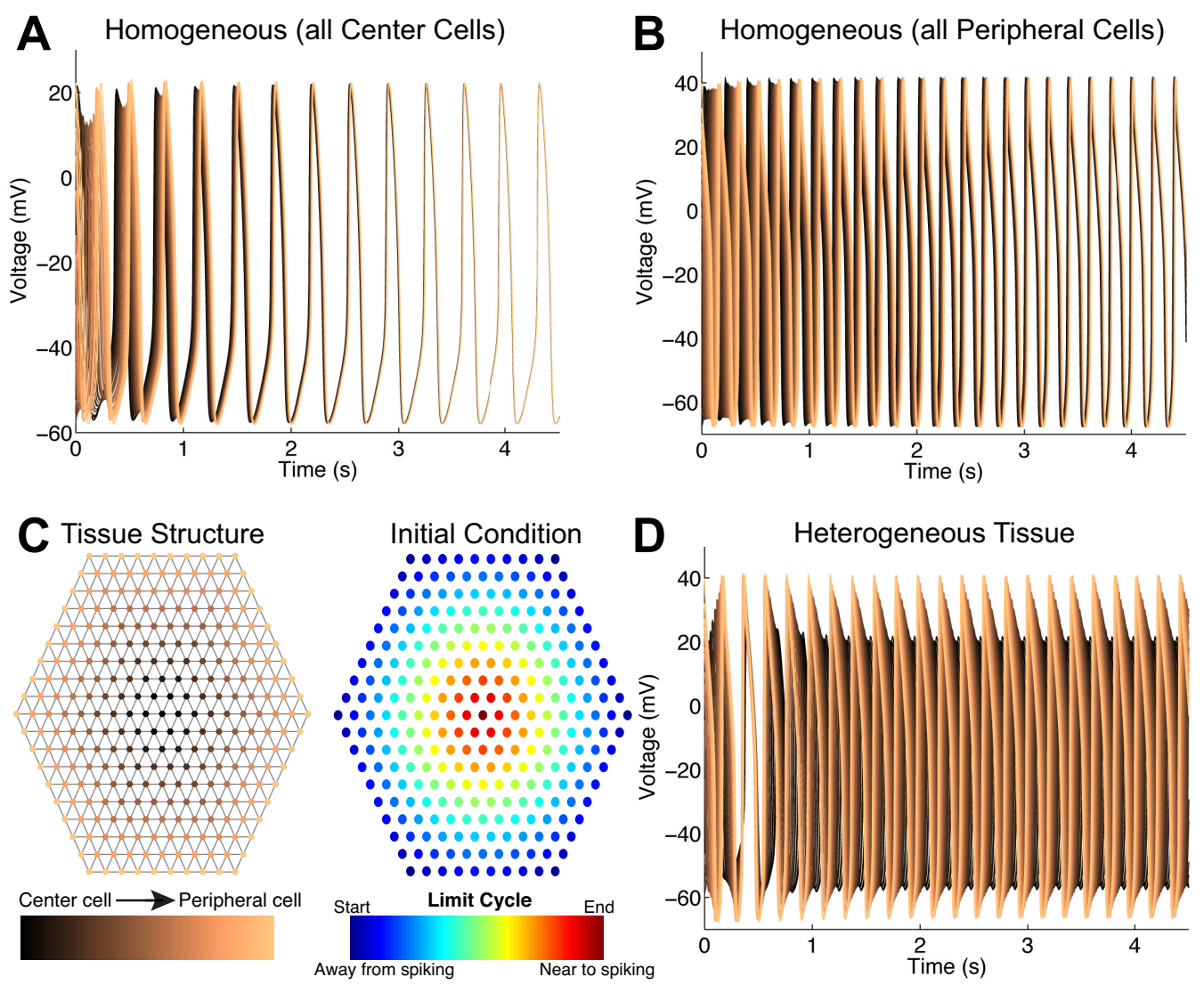

Figure 2: Simulation results of the full pacemaker model. The color-coding schemes in $\mathbf{A}$ and $\mathbf{B}$ do not represent intrinsically different cell types, but are chosen to show spatial location. A) With identical cells (all center): after an initial transient of center-to-peripheral traveling wave, the system settles to complete synchrony. B) With identical cells (all peripheral), the transient center-to-peripheral wave lasts longer and has larger frequency than the center cells in $\mathbf{A}$, but eventually settles to complete synchrony. C) Left) the architecture is nearest neighbor coupling (gray lines connecting dots) used for all tissue simulations, the color coding indicates different cell types in the heterogeneous tissue used in D. There are 271 total pacemaker cells on a hexagonal grid with all connected cells equidistant apart. C) Right) the same initial condition for all tissues where cells near the center are closer to spiking, and peripheral cells are gradually further away from spiking; this sets the tissue to transiently have traveling waves originating in the center. D) In a completely heterogeneous tissue, the transient (center-to-peripheral wave) is very brief, after which the system settles to a persistent traveling wave solution that originates in the periphery and ends in the center. In all panels, coupling strength was constant $g_{\text {gap }}=0.25 \mathrm{nS}$.

We next performed simulations of the SAN tissue models. Simulations of the SAN tissue presented with a variety of spatio-temporal dynamics that depend on coupling strength and cell distribution (heterogeneous or homogeneous). To investigate the role of heterogeneity in the SAN tissue, we simulate a homogeneous tissue comprised of either all center (Fig. 2A) or all peripheral (Fig. 23) cells, or a heterogeneous tissue with a transition from center to peripheral cell 
types (Fig. 2D). Experimental observations suggest that pacemaking originates in the center of the SAN tissue. Consistent with these conditions, for all three tissue structures, we set the initial condition of all 31 state variables, such that center cells were "closer" to spiking and peripheral cells were "further" from spiking, in a gradual monotonic way (Fig. 2 C right). This resulted in an initial but transient traveling wave that originated in the center for all three tissues. Closer inspection of the voltage traces reveals that the transient dynamics have different durations; in particular, the transient persists longest in the homogeneous peripheral cell tissue, followed by the homogeneous center cell tissue, with the heterogeneous tissue having the shortest transient before settling to the steady-state wave solution. Interestingly, in both homogeneous cases, after an initial transient time period, electrical activity of all of SAN cells became highly synchronized. However, in the heterogeneous SAN tissue (Fig. $2 \mathrm{C}$ left), a brief transient period is followed by a stable traveling wave, originating in the periphery (Fig. 2D), consistent with findings by Oren and Clancy (2010). Note that the coupling strengths are the same for all connected cells and in all three tissues $\left(g_{\text {gap }}=0.25 \mathrm{nS}\right)$. Among other tissue attributes, transient times depend on initial conditions; here we chose the initial condition primed for central wave generation in all large-scale simulations that did not correspond to the steady-state traveling wave for any of the tissue structures.

We did not exhaustively vary the coupling strengths $g_{g a p}$ in each tissue structure because of computational resources, but we did simulate each tissue structure with several sets of gap junction values. As coupling strength increases in a given tissue, the transient times generally decrease, as one might intuitively expect due to stronger interactions between cells (see Fig. 7 for the same tissues as Fig. 2 but with larger $g_{g a p}$ ). Although this is only apparent by inspection, we will show this is theoretically true in the next section with the reduced phase model theory.

Ultrastructure studies of the SAN tissue suggest that there may be an increase in gap junctional density from the center to the periphery (Masson-Pévet et al., 1979, Bleeker et al., 1980; Boyett et al. 2000). However, histology also illustrates the irregular structure within SAN tissue, consisting of discontinuous myofibrils, fat, and fibrous tissue (Li et al., 2015, Csepe et al., 2016), suggesting a wide range of possible cell-cell coupling within the SAN tissue. Therefore, in addition to considering tissues with fixed gap junction coupling strength, we also consider tissue in which $g_{\text {gap }}$ varies depending on cell-type and location. We consider 2 types of tissues: i) a linear gradient where peripheral cells have $g_{\text {gap }} 15 \times$ 's stronger than center cells (termed Strong Peripheral Gap Junction, see Oren and Clancy (2010) who also considered this), ii) a linear gradient where center cells have $g_{g a p} 15 \times$ 's stronger than peripheral cells (termed Strong Central Gap Junction). In total, we have three different types of coupling schemes (constant $g_{g a p}$ and two types of linear gradient $g_{g a p}$ ) that are not directly comparable to each other. We chose these tissues to assess the robustness of our observations, and importantly to test the robustness of the subsequent reduced phase model theory in the next section.

Although there are significant differences in the three types of coupling schemes (constant $g_{\text {gap }}$, Strong Peripheral Gap Junction, Strong Central Gap Junction), the following trends hold:

- The only steady-state traveling wave solution we observe in homogeneous tissue (center or peripheral) is complete synchrony, with our initial conditions.

- The only observed steady-state traveling wave in the heterogeneous tissue are ones that originate in the periphery and end in the center (see Kirchhof et al. (1987) for experimental evidence of peripheral-to-center wave in the rabbit SAN when the atrium is removed). In a given tissue, the frequency of this traveling wave decreases with gap junction value(s). 
- For the same initial condition (Fig. $2 \mathrm{C}$ right), we observed that: the center-to-peripheral traveling wave transient persists longest in the homogeneous peripheral cell-type tissue, with the heterogeneous tissue generally having the shortest transient times - there are some exceptions where the homogeneous center cell-type tissue has shorter transient times than the heterogeneous tissue (especially with larger $g_{g a p}$ values), but the transient times for these two tissues are very similar in those cases.

These results will be substantiated with plots of the large-scale simulations and characterized with the reduced phase model in the next section. Specific voltage traces of the large-scale model for various tissue types, coupling schemes $g_{\text {gap }}$ values, etc., are shown in Figs. 7 f 9 and Figs. S3-S5 (S1Text.pdf).

\subsection{Reduced Phase Model Analysis of Traveling Waves}

To more clearly understand the observations in the large-scale simulations, we use a reduced phase model framework. This will not only make mathematical analysis of the tissue dynamics feasible, but will also provide a framework for future investigations of large-scale tissue models of SAN tissue. The subsequent analysis relies on computing various functions from the individual Severi cell models, but did not rely on the computationally expensive step of simulating the large-scale tissue model.
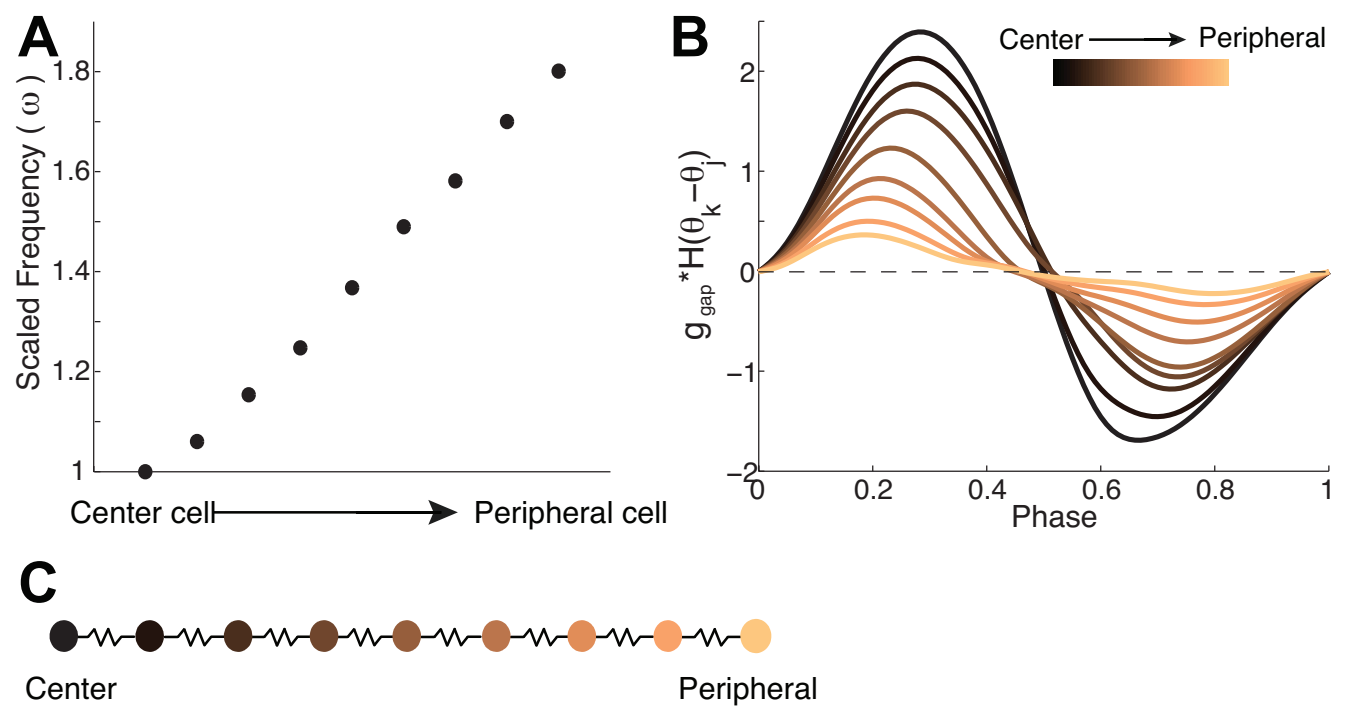

Figure 3: The phase oscillator model approximation to the large-scale Severi pacemaker model. A) The intrinsic frequencies were obtained from the full model and scaled by the largest period. B) The interaction functions $H_{j, k}$ are approximated with $H_{j}$ for $k=j-1$ and $k=j+1$; see Eq $\left[7\right.$. We set $g_{\text {gap }}=1 \mathrm{nS}$ for illustration purposes. C) The 1D chain approximation to the full 2D model.

We use the phase reduced models (see Methods) to approximate the behavior of the largescale SAN tissue, where each cell type has an equation of the form:

$$
\frac{d \theta_{j}}{d t}=\omega_{j}+\sum_{k} H_{j, k}\left(\theta_{k}-\theta_{j}\right)
$$


The frequencies $\omega_{j}$ are all scaled by the slowest intrinsic frequency (often the center cell with $T=0.353 \mathrm{~s}$ ), such that in the heterogeneous tissue, the center cells have $\omega=1$ and peripheral cells $\omega \approx 1.8$ (see Fig. $3 \mathbf{A}$ for the full range of possible $\omega$ values and Fig. 3B for unscaled versions of $H_{j, k}$ ). Note that the intrinsic period of the peripheral cell is $T=0.196 \mathrm{~s}$.

The reduced phase model consists of a 1D chain of 9 phase oscillators with nearest neighbor coupling: $H_{j, k} \equiv 0$ unless $k=j-1$ or $k=j+1$ (see Fig. 3 C), such that Eq. (12) for this 1D chain is re-written as:

$$
\begin{aligned}
\frac{d \theta_{1}}{d t} & =\omega_{1}+H_{1,2}\left(\theta_{2}-\theta_{1}\right) \\
\frac{d \theta_{j}}{d t} & =\omega_{j}+H_{j, j-1}\left(\theta_{j-1}-\theta_{j}\right)+H_{j, j+1}\left(\theta_{j+1}-\theta_{j}\right), \text { for } j=2, \ldots, 8 \\
\frac{d \theta_{9}}{d t} & =\omega_{9}+H_{9,8}\left(\theta_{8}-\theta_{9}\right)
\end{aligned}
$$

This model is amenable to analysis of traveling wave dynamics (Kopell and Ermentrout, 1986, Ermentrout and Terman, 2010, Keener and Sneyd, 1998). Traveling wave solutions to this system are of the form

$$
\theta_{j}(t)=\Omega t+\xi_{j}
$$

where $\Omega$ is the ensemble (scaled) frequency of the coupled network and $\xi_{j}$ represents the phase lags of each cell type (i.e., $\xi_{j}=0$ for all $j$ is complete synchrony). Substituting Eq. (14) into Eq. 13] gives:

$$
\Omega=\omega_{j}+\sum_{k} H_{j, k}\left(\xi_{k}-\xi_{j}\right), \text { for } j=1, \ldots, 9 .
$$

The system of 9 nonlinear equations and 10 unknowns $\left(\Omega, \xi_{j}\right)$ can be reduced to 9 unknowns by exploiting the periodicity of $\xi_{j} \in[0,1)$, and setting $\xi_{1}=0$. Thus, if $\xi_{j}-\xi_{j+1}>0$ for $j=2, \ldots, 8$ the traveling wave originates at the center $(j=1)$, and if $\xi_{j+1}-\xi_{j}>0$ the traveling wave originates in the periphery (see Ermentrout and Terman (2010) for similar exposition) 2

Traveling wave solutions to the reduced phase model system exist (numerically) whenever the variables $(\Omega, \vec{\xi})$ satisfy the Eqs. $(14)-(15)$. For each of the coupling schemes and all three tissue types ( 2 homogeneous, uniform heterogeneous), we found several sets of $g_{\text {gap }}$ values where traveling wave solutions numerically existed. Solutions to Eqs. (14)-(15) were found using fmincon in MATLAB with high precision (agreement of at least 3 decimal places in each equation).

To address the stability of traveling wave solutions when they exist, we rely on a theorem by Kopell and Ermentrout (1986); Ermentrout (1992) which provides sufficient conditions for the stability of traveling wave solutions. The theorem re-stated for these reduced phase model networks is:

Theorem 1. The traveling wave solution $(\Omega, \vec{\xi})$ that satisfies Eq. $\sqrt{15})$ is stable if the coupled network is irreducible and $H_{j, k}^{\prime}\left(\xi_{k}-\xi_{j}\right) \geq 0$.

The proof is technical and can be found in Kopell and Ermentrout (1986); Ermentrout (1992). We prove the stability of traveling waves by applying this theorem to a range of parameter sets in the 3 tissue types and coupling regimes. Note that the chain of oscillators (Fig. $3 \mathrm{C}$ ) is irreducible

\footnotetext{
${ }^{2}$ The exception is if the traveling wave solution has very fast frequency and the next wave starts before the current one ends, in which case the sign of $\xi_{j+1}-\xi_{j}$ is different for a $j$, see Fig $10 \mathbf{B}$ in yellow and Fig $11 \mathrm{D}, \mathbf{E}, \mathbf{F}$.
} 

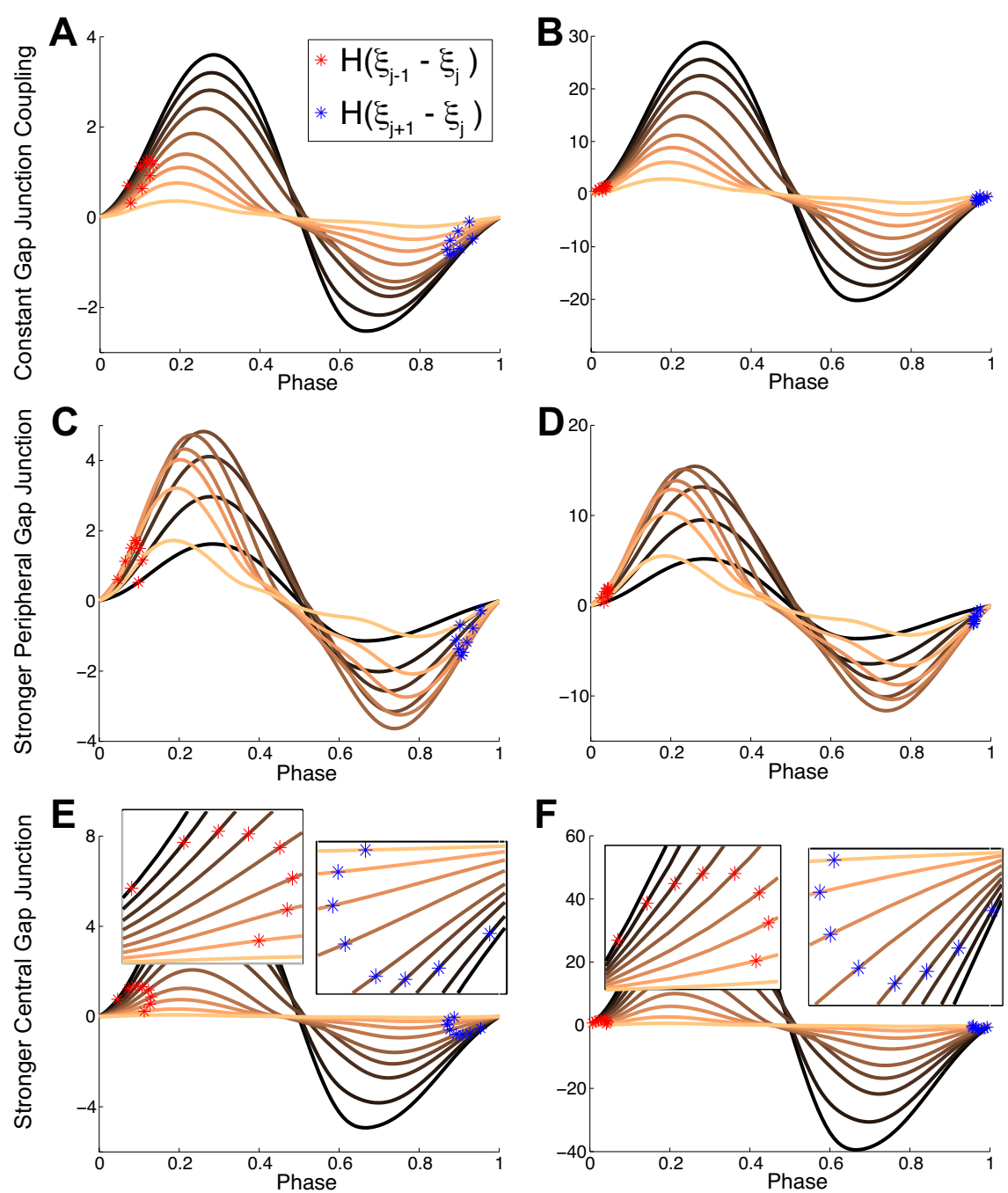

Figure 4: Existence and stability of traveling waves for heterogeneous tissues. The following plots show the $H$-functions for a specific gap junction coupling strengths, along with calculated quantities $H_{j, k}\left(\xi_{j-1}-\xi_{j}\right)$ and $H_{j, k}\left(\xi_{j-1}-\xi_{j}\right)$. A sufficient condition for stability of the waves relies, in part, on $\frac{d H}{d \theta}\left(\xi_{j-1}-\xi_{j}\right)>0$ and $\frac{d H}{d \theta}\left(\xi_{j+1}-\xi_{j}\right)>0$ (see main text), which holds for all tissues here. In all the regimes we explored with heterogeneous cells, traveling waves originating in peripheral cells exist and are stable. A) For constant gap junction coupling, with $g_{g a p}=0.25 \mathrm{nS}$ in the full largescale tissue. B) Same as A but with larger $g_{\text {gap }}=2 \mathrm{nS}$. C) Gap junction strength $g_{\text {gap }}$ varying monotonically from center to peripheral, with largest value at the peripheral cells $\left(g_{\text {gap }}=1.25 \mathrm{nS}\right)$ and smallest value at center cells $\left(g_{\text {gap }}=\right.$ $1.25 / 15 \mathrm{nS}$ ). D) Same as $\mathbf{C}$ but with much larger coupling, $g_{\text {gap }}=4 \mathrm{nS}$ at peripheral cells and $g_{\text {gap }}=4 / 15 \mathrm{nS}$ at center cells. E) Gap junction strength varying monotonically, with largest at the center cells $\left(g_{\text {gap }}=0.5 \mathrm{nS}\right)$ and smallest at peripheral cells $\left(g_{\text {gap }}=0.5 / 15 \mathrm{nS}\right)$. The 2 insets are zoomed-in pictures to convincingly show that the stars are where $H$ is increasing (i.e., $\frac{d H}{d \theta}>0$ ). F) Same as $\mathbf{E}$ but with much larger coupling, $g_{\text {gap }}=4 \mathrm{nS}$ at center cells and $g_{\text {gap }}=4 / 15 \mathrm{nS}$ at peripheral cells. 
since every oscillator is connected, so the only condition to check is $H_{j, k}^{\prime}\left(\xi_{k}-\xi_{j}\right) \geq 0$. In correspondence with the large-scale tissue model, recall that we consider: i) constant gap junction coupling, and gradient gap junction coupling with ii) a linear gradient where peripheral cells have $g_{\text {gap }}$ 15×'s stronger than center cells (termed Strong Peripheral Gap Junction), and iii) a linear gradient where center cells have $g_{g a p} 15 \times$ 's stronger than peripheral cells (termed Strong Central Gap Junction).

Synchrony is the only periodic steady state for the homogeneous tissues (all center cells or all peripheral) in the phase models; we explain why this state is always stable. With complete synchrony, $\xi_{j}=0$ for all $j$ in Eq. (14, so we only have to check that $H_{j, k}^{\prime}(0) \geq 0$ according to the theorem. This is easily verified in Fig. $3 \mathbf{B}$, and holds for any $g_{\text {gap }}>0$ because it simply scales the the curve, as well as any other homogeneous tissue with any one of the 9 cell types. Thus, complete synchrony is always a stable state in the homogeneous tissues we consider.

In the heterogeneous tissues, the traveling wave solutions consist of $\xi_{j}>0$, so we have to verify that $H_{j, k}^{\prime}\left(\xi_{k}-\xi_{j}\right) \geq 0$ numerically to show that the solution is stable. We initially considered 15 total parameter sets for the large-scale heterogeneous tissues; we discovered traveling wave solutions existed in 14 (5 with constant $g_{g a p}$ coupling, 4 with strong peripheral, 5 with strong central), and in all these cases, the traveling waves are stable. We discuss the parameter set without a traveling wave solution below. Fig. 4 shows the smallest (left column) and largest coupling values (right column) considered, with each coupling schemes organized by row. Notice that in all instances, $H_{j, k}^{\prime}>0$, indicating that the traveling wave is stable. Stability of all other heterogeneous parameter sets are shown in Fig. S1 (S1Text.pdf).

The coupled reduced phase model can also qualitatively reproduce the key property of tissue frequency from the large-scale model in both uniformly coupled homogeneous (either all center or all peripheral) or heterogeneous population of SAN cells. This is demonstrated with a variety of coupling schemes and values: with constant gap junction (Fig. 5A), strong peripheral gap junction (Fig. 5B ), strong central gap junction (Fig. 5C). The solid lines in Fig. 5 are the $\Omega$ from the reduced phase model, using Eq. (14) but scaled by the smallest intrinsic frequency to have units of $(1 / \mathrm{sec})$, while the tissue frequencies from the large-scale model (stars) are calculated by taking the average of the peak frequencies of the power spectrums of the voltage (in the last $10 \mathrm{~s}$ out of $20 \mathrm{~s}$ of biological time) for all 271 pacemaker cells.

Although the matches between the curves (phase model) and stars (large-scale simulations) in Figure 5 are not quantitatively accurate for heterogeneous tissues, the qualitative trend that wave frequency decreases with gap junction strength is captured. The comparisons deviate most with strong peripheral (gradient coupling strength, Fig. 53 B), and are most accurate with strong central (gradient coupling strength, Fig. 5 $\mathrm{C}$ ); the match with constant gap junction coupling in Figure $5 \mathbf{A}$ is better than $\mathbf{B}$ and worse than $\mathbf{C}$. This is all likely due to the violation (or conformity) of the weak heterogeneity assumption in the reduced phase model. This assumption is severely violated with stronger peripheral gap junction because the larger number of peripheral cells with the hexagonal grid (Fig. $2 \mathrm{C}$ left) combined with larger $g_{g a p}$ results in a phase model with large heterogeneity from cell-type to cell-type (cell-types are grouped and averaged in the reduced phase model). Recall that the intrinsic frequency difference varies greatly by almost a factor of 2 $(\approx 1.8 \times)$. Conversely, with stronger central gap junction, the larger number of peripheral cells is counter balanced by weaker $g_{g a p}$, resulting in weaker heterogeneity from cell-type to cell-type. Note that the deviations in wave frequency are less than $1 \mathrm{~Hz}$ but that the intrinsic frequencies differ by $2.27 \mathrm{~Hz}$.

Finally, since the dynamics of the large-scale pacemaker tissue model only transiently reproduce the physiologically-observed center-to-peripheral wave pattern, one possible mechanism 

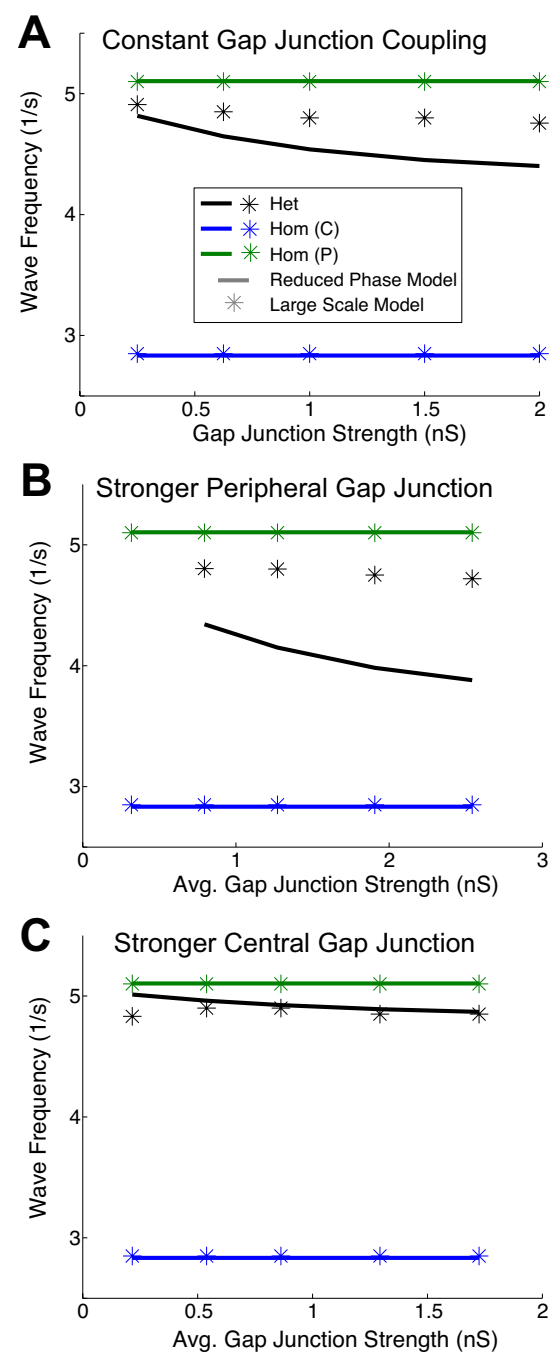

Figure 5: Reduced phase model theory captures the traveling wave frequency in the large-scale model. The ensemble traveling wave frequency in the reduced phase model ( $\Omega$ in Eq. (14) scaled to $1 / \mathrm{sec}$ ) plotted with solid lines, is compared to the traveling wave frequency of the full large-scale coupled tissue model (stars). Here we only consider the reduced phase models that correspond to the parameter sets we initially considered in the large-scale models. In all panels, the periodic steady state for both homogeneous Center (blue) and Peripheral (green) cell tissues is complete synchrony; in the heterogeneous tissue (black), the wave originates in the periphery and terminates somewhere near the center. A) For constant gap junction coupling, varying $g_{\text {gap }}$ from $0.25 \mathrm{nS}$ to $2 \mathrm{nS}$ in the full large-scale tissue. B) Gap junction strength $g_{\text {gap }}$ varying monotonically from center (weakest) to peripheral (strongest); the x-axis shows the average $g_{\text {gap }}$ among all connected cells for a given tissue configuration. In the heterogeneous tissue, a traveling wave solution does not exist for smaller $g_{g a p}$. C) Gap junction strength $g_{g a p}$ varying monotonically from center (strongest) to peripheral (weakest); $\mathrm{x}$-axis has the same convention as $\mathbf{B}$.

explaining this response is that autonomic regulation may regularly reset "initial conditions" to drive the center-to-peripheral wave. Therefore, we sought to quantify the characteristics of 
the transient dynamics, before the coupled system settles to a stable traveling wave, as well as the agreement between the large-scale simulations and reduced model. A perturbation $\vec{\eta}(t)$ to a traveling wave solution $\vec{\theta}(t)=\Omega t+\vec{\xi}$ is:

$$
\theta_{j}(t)=\Omega t+\xi_{j}+\eta_{j}(t)
$$

the transient dynamics can be approximated by assuming perturbations are small $\left(\eta_{j}=O(\varepsilon)\right.$ for all $j$ ); see Methods section for further details. This equation in matrix-vector form is (see equation (11):

$$
\frac{d \vec{\eta}}{d t}=A \vec{\eta}
$$

When a traveling wave solution is stable, all but one of the eigenvalues of $A$ have negative real part; there is a a distinct zero eigenvalue $\lambda_{0}=0$ with eigenvector $\overrightarrow{1}$ that corresponds to a constant shift in the traveling wave solution - a constant shift in all variables is a perturbation that does not decay because the result is the same traveling wave solution.

Recall that the vector of perturbations can be approximated by using an eigenvector expansion (see Methods section). Let the eigenvectors of $A$ be $\vec{\psi}_{j}$ with eigenvalues $\lambda_{j}$ : $A \vec{\psi}_{j}=\lambda_{j} \vec{\psi}_{j}$. We use the initial condition from the large-scale (Fig. 2 $\mathrm{C}$ left) mapped to the phase variable; this is well-approximated by $\vec{\eta}_{0}(j)=1-j * \frac{1}{10}$ (traveling wave starting at center) but we use the actual mapped phase value. The vector of perturbations $\vec{\eta}$ is approximated via:

$$
\vec{\eta}(t)=\left(\vec{\psi}_{1}, \overrightarrow{\eta_{0}}\right) e^{\lambda_{1} t} \overrightarrow{\psi_{1}}
$$

where $\lambda_{1}$ is the eigenvalue with large nonzero real part, and $(\vec{v}, \vec{w})$ is the usual inner product. In the Supplementary Material (S1Text.pdf), we show that this solution is numerically the same as the full eigenvector expansion (see Fig. S2 in S1Text.pdf). The times to the steady-state traveling wave solutions are approximated by determining when the perturbations decay to a specified tolerance: $\|\vec{\eta}(t)\|<\epsilon$. In all figures $\left[6 \mathbf{A}, \mathbf{C}, \mathbf{E}, 7,99\right.$, we use $\epsilon=1 \times 10^{-4}$.

To numerically determine the time point at which the transients have decayed in the largescale simulations, we check that the time course of the voltage in a period $T_{c}$ (inverse of frequency calculated in Fig. 5(stars)) is within a specified tolerance of the next cycle; the transient time is over only when the voltages match for all 271 cells:

$$
\arg \min _{t}\left\{\frac{\left|v_{j}(t)-v_{j}\left(t+T_{c}\right)\right|}{M_{k}}<\mathbf{e}\left|\max _{t}\left(v_{j}(t)\right)-\min _{t}\left(v_{j}(t)\right)\right|, \quad j=1, \ldots, 271 \text { cells }\right\}
$$

where $M_{k}=$ number of time points in a period, and $\mathbf{e}=1 \times 10^{-3}$ for the two homogeneous tissue and $\mathbf{e}=1.7 \times 10^{-3}$ for all heterogeneous tissue ${ }^{3}$ (black stars). See Figure 6A, $\mathbf{C}, \mathbf{E}$ for comparisons of the transient times.

We remark that numerically calculating a precise time when the large-scale model reaches a steady-state traveling wave solution is tenuous, because the results are sensitive to the tolerance. Yet despite this, we find great qualitative matches between the reduced phase model and the large-scale tissue with relatively generic tolerances. Indeed, Figure 6A, C, E shows the match

\footnotetext{
${ }^{3}$ This tolerance was the minimum that gave reasonable results when incrementing by $1 \times 10^{-4}$ from $\mathbf{e}=1 \times 10^{-3}$. One data point corresponding to strong central coupling in the heterogeneous tissue and small $\frac{0.5}{15} \mathrm{nS} \leq g_{\text {gap }} \leq 0.5 \mathrm{nS}$ (Fig. 6C black star) did not yield reasonable transient times for similar valued tolerances; see vidx_Het_g2_s2.mp4 in S2.zip.
} 

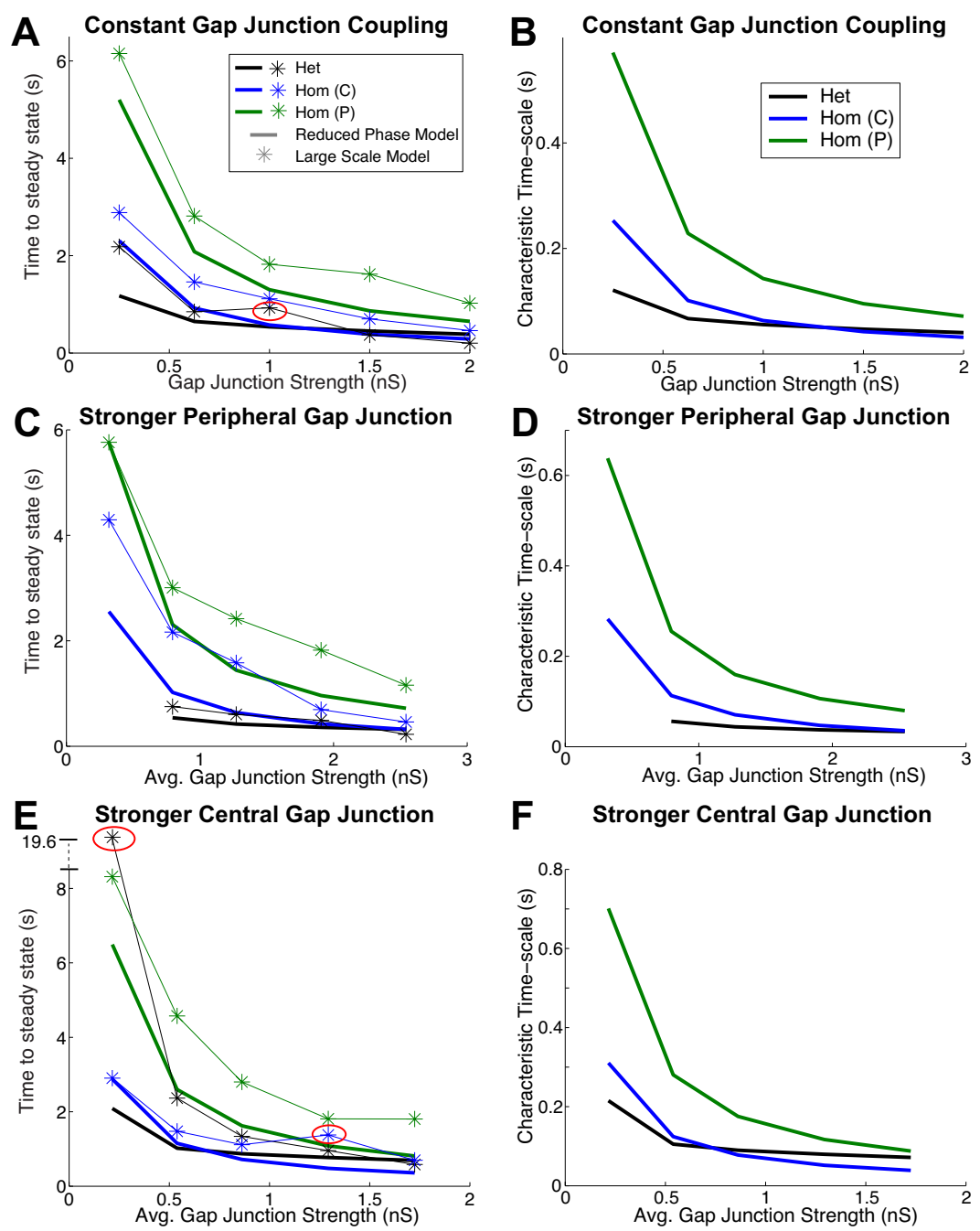

Figure 6: Reduced phase model theory captures the transient times before settling to steady-state traveling waves. Left Column: for a given tissue configuration with initial condition primed to center-to-peripheral traveling waves (Fig. $2 \mathrm{C}$ right), the transient times from largest to smallest are generally: homogeneous peripheral cell tissue (green), homogeneous center cell tissue (blue), heterogeneous tissue (black). As coupling strength increases, transient times decrease and the differences between blue and black are diminished. Although the match between large-scale tissue (stars) and reduced phase model (solid curves) is not always precise, the qualitative trends are captured. The right column shows the characteristic time-scale $\left(\left(\left|\lambda_{1}\right|\right)^{-1}\right.$, where $\lambda_{1}$ is the largest negative eigenvalue of $A$; see Eq. 10 but rescaled to seconds), which is a measure of transient time that does not depend on initial condition or numerical tolerance. A, B) For constant gap junction coupling, varying $g_{\text {gap }}$ from $0.25 \mathrm{nS}$ to $2 \mathrm{nS}$ in the full large-scale tissue. C, D) Gap junction strength $g_{\text {gap }}$ varying monotonically from center (weakest) to peripheral (strongest); the x-axis shows the average $g_{\text {gap }}$ among all connected cells for a given tissue configuration. E, F) Gap junction strength $g_{g a p}$ varying monotonically from center (strongest) to peripheral (weakest); $\mathbf{x}$-axis has the same convention as $\mathbf{C}, \mathbf{D}$. The 3 red ovals are when the effects from the boundaries of the hexagonal grid persist so that the 1D chain is is a bad approximation - see Supplementary Material (from top to bottom: vidx_Het_g3_s3.mp4, vidx_Het_g2_s2.mp4, vidx_HomC_g33_s3.mp4 in S2.zip). 
between phase model (thick lines) and large-scale tissues (stars connected by lines) can be accurate. When the match is not precise, the following trends are captured: homogeneous peripheral cells (green) have longer transients, followed by homogeneous center (blue), then heterogeneous (black); transient times decrease with coupling strength; homogeneous center and heterogeneous tissues have similar transient time with stronger coupling. The 3 red ovals in Fig. 6 left column are for conditions for which boundary effects (i.e., corners of hexagonal grid) influence transient times (see Supplementary Material referenced in Fig. 6 caption). Because our grid is truly 2-dimensional (as opposed to radially symmetric grids where nearest neighbor coupling spans disparate Euclidean distances), such boundary effects cannot easily be removed.

A common way to quantify the duration of transient dynamics is to consider the characteristic time-scale, which for a linear system is defined as the inverse of the modulus of the eigenvalue with largest (negative) real part $\left|\lambda_{1}\right|^{-1}$ of $A$. This transient time measure does not depend on a particular initial condition or numerical tolerance. The characteristic time-scale from the reduced phase model is shown in the right column of Fig. $6 \mathbf{B}, \mathbf{D}, \mathbf{F}$, and it does indeed capture all of the trends in both the reduced phase model and large-scale tissues: homogeneous peripheral cell tissue has the longest transients, transient time decreases as coupling strength increases, heterogeneous tissue has the shortest transient time with weaker coupling, homogeneous central cell tissue has similar transient times as the heterogeneous tissues with stronger coupling.

The results in Figure 6 are further detailed with plots of the voltage trajectories, along with the transient times calculated both via the reduced phase model (cyan arrows, Figs. 749) and large-scale simulation (blue arrows, Figs. 7f 9). In Figures 7,9 , it is apparent that both transient time estimations from the the reduced phase model and the large-scale simulations are relatively accurate. The only exceptions are perhaps when the large-scale simulations exhibit behavior that cannot be well-approximated by a 1D chain of oscillators (i.e., plots that correspond to the 3 red ovals in Fig. 6 left column). The constant gap junction coupling scheme is shown in Figure 7 with each row consisting of a particular cell distribution (i.e., heterogeneous or homogeneous) and each column has the same set of $g_{g a p}$ values. We again see that for a given coupling scheme (i.e., column) the homogeneous peripheral cell tissue has the longest transient times, generally followed by the homogeneous center cell tissue, and then the heterogeneous tissue. Figure 8 shows the gradient $g_{\text {gap }}$ with stronger peripheral coupling, and Figure 9 is with stronger central coupling. See Figures S3-S5 (S1Text.pdf) for other parameter values not shown in Figures 749

Thus far, we have compared the large-scale models with its corresponding reduced phase model. We now use the phase model to investigate the spatio-temporal dynamics for the heterogeneous networks in a large region of parameter space. For all 3 coupling schemes, we vary both the initial condition and gap junction coupling values. We consider 21 initial conditions varying gradually, i.e., where the system starts primed for center-to-peripheral traveling waves (Fig.10. i in gray inset), to starting at complete synchrony (Fig 10, iii), to being primed for peripheral-tocenter traveling waves (Fig $10 \mathrm{v}$ ). We choose 391 gap junction coupling values (or sets) that span a large range (Verheule et al. 2001). The black stars correspond to the large-scale Severi model simulations that have been investigated. Note that simulation of the large-scale pacemaker tissue model over this wide parameter regime for gap junctional values and initial conditions would require a prohibitively large amount of computational resources.

In this study of the reduced model, we first determined if the system settled to a periodic solution (i.e., traveling wave that satisfies Eqs. 13 - 15). With constant gap junction coupling (Fig 10A) and with stronger central gap junction (Fig 10 C), traveling wave solutions exist and are peripheral-to-center (green) for all $g_{g a p}$ and initial conditions considered. With stronger peripheral gap junction (Fig $[10 \mathrm{~B}$ ), peripheral-to-center traveling waves exist for almost all parameters, 
Constant Gap Junction Coupling $\mathbf{T}$ Transient time (large-sims) Transient time (theory, phase model)
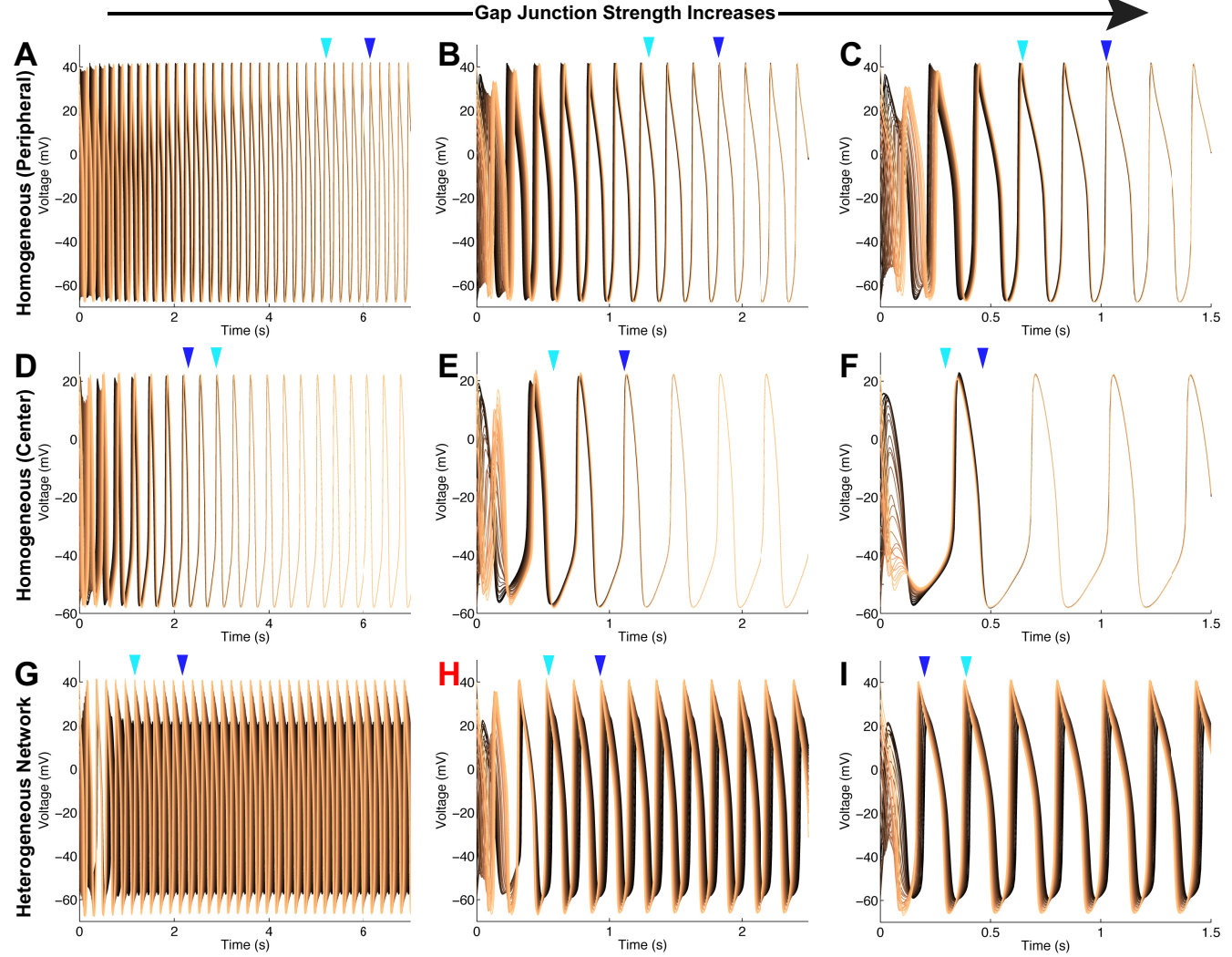

Figure 7: Voltage trajectories in large-scale model plotted with the theoretical transient time (reduced phase model) and large-scale transient time: with constant gap junction coupling. By inspection, the transient times from the reduced phase model (cyan arrow) and large-scale model (blue arrow) are reasonable. Each column has different coupling strengths, each row is a particular type of tissue. Left column $(\mathbf{A}, \mathbf{D}, \mathbf{G})$ is with $g_{\text {gap }}=0.25 \mathrm{nS}$. Middle column $(\mathbf{B}, \mathbf{E}, \mathbf{H})$ is with $g_{\text {gap }}=1 \mathrm{nS}$. Right column $(\mathbf{C}, \mathbf{F}, \mathbf{I})$ is with $g_{\text {gap }}=2 \mathrm{nS}$. A)-C) The homogeneous tissue of peripheral cells. D)-F $)$ The homogeneous tissue of center cells. G)-I) The heterogeneous tissue consisting of 9 different cell types. In each column, the transient generally last longest for homogeneous peripheral cell tissue (top row), following by homogeneous center cells, with the heterogeneous tissue having the shortest transient. The label $\mathbf{H}$ is red to correspond with the red oval in Figure 6 A. 
Stronger Peripheral Gap Junction

$\nabla$ Transient time (large-sims) Transient time (theory, phase model)

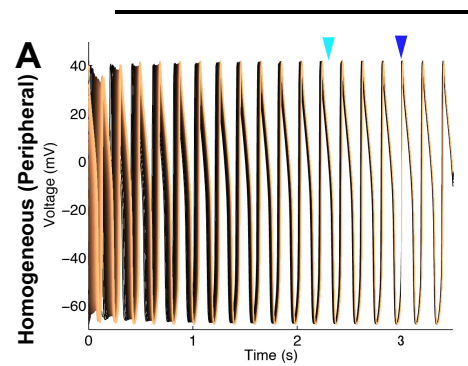

$B_{40}$
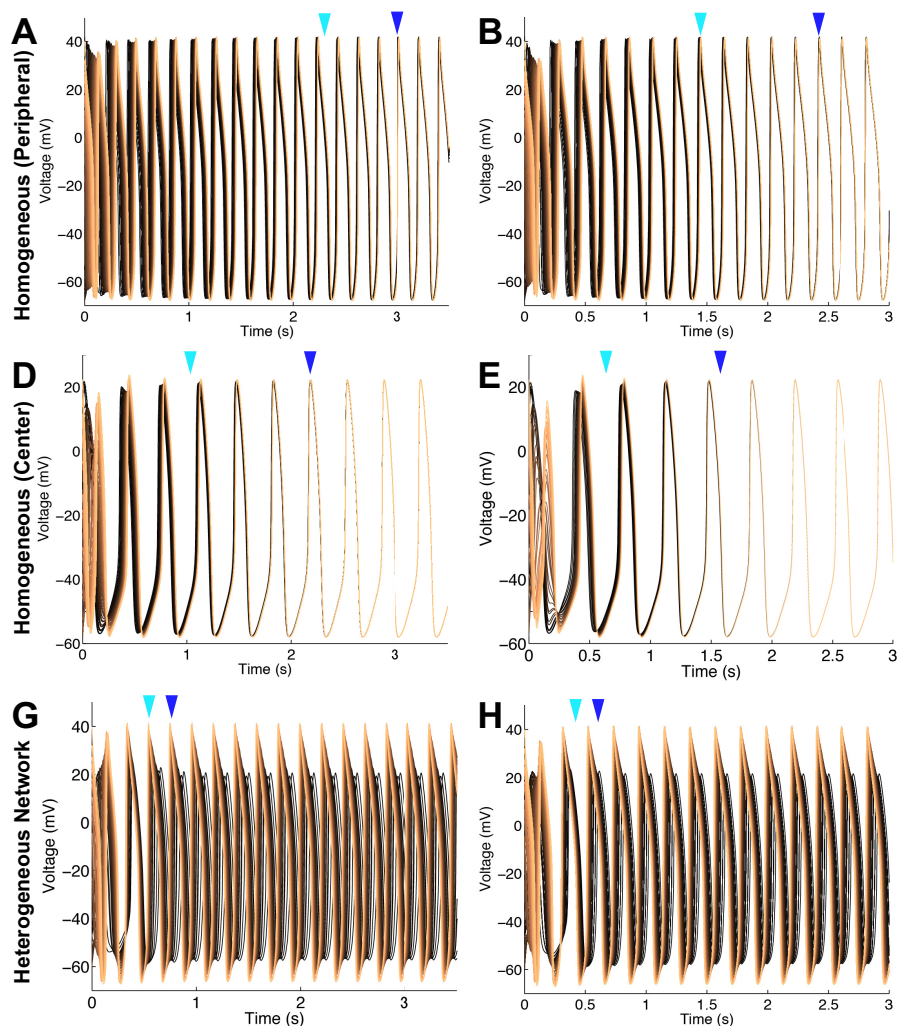

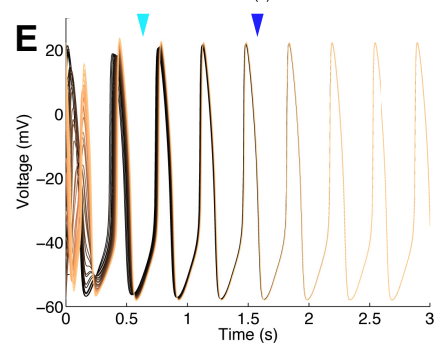

$\mathbf{H}_{40}$.

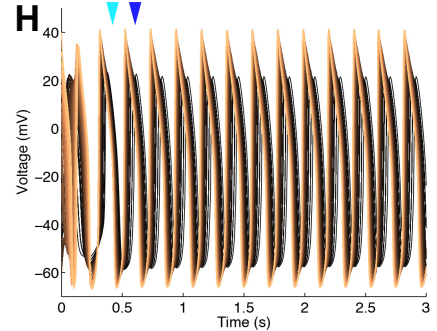

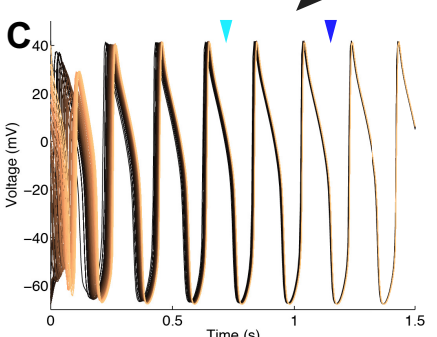
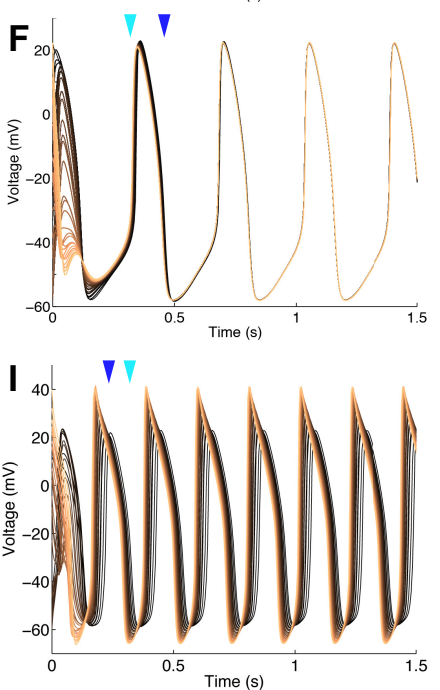

Figure 8: Voltage trajectories in large-scale model plotted with the theoretical transient time (reduced phase model) and large-scale transient time: with strong peripheral coupling. Again by inspection, the transient times from the reduced phase model (cyan arrow) and large-scale model (blue arrow) are reasonable. Each column has different coupling strengths, each row is a particular type of tissue (same format as Fig. 7). Left column (A, D, G) is with $\frac{0.625}{15} \mathrm{nS} \leq g_{\text {gap }} \leq 0.625 \mathrm{nS}$. Middle column $(\mathbf{B}, \mathbf{E}, \mathbf{H})$ is with $\frac{2}{15} \mathrm{nS} \leq g_{\text {gap }} \leq 2 \mathrm{nS}$. Right column $(\mathbf{C}, \mathbf{F}, \mathbf{I})$ is with $\frac{4}{15} \mathrm{nS} \leq g_{\text {gap }} \leq 4 \mathrm{nS}$. In each column, the transient generally last longest for homogeneous peripheral cell tissues (top row), following by homogeneous center cells, with the heterogeneous tissues having the shortest transient. 


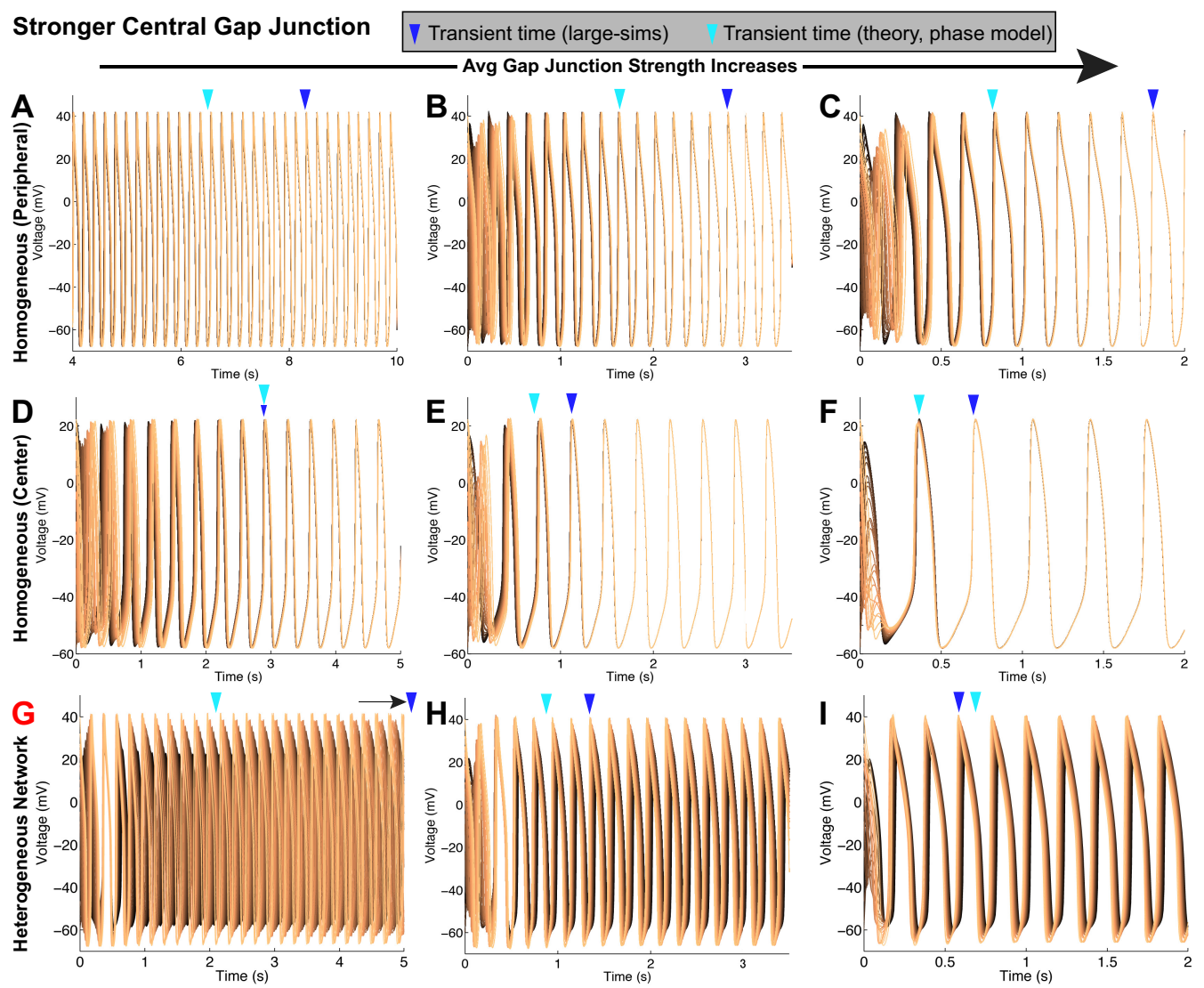

Figure 9: Voltage trajectories in large-scale model plotted with the theoretical transient time (reduced phase model) and large-scale transient time: with strong central coupling. Again by inspection, the transient times from the reduced phase model (cyan arrow) and large-scale model (blue arrow) are reasonable. Each column has different coupling strengths, each row is a particular type of tissue (same format as Fig. 7). Left column $(\mathbf{A}, \mathbf{D}, \mathbf{G})$ is with $\frac{0.625}{15} \mathrm{nS} \leq g_{\text {gap }} \leq 0.625 \mathrm{nS}$. Middle column $(\mathbf{B}, \mathbf{E}, \mathbf{H})$ is with $\frac{2}{15} \mathrm{nS} \leq g_{\text {gap }} \leq 2 \mathrm{nS}$. Right column $(\mathbf{C}, \mathbf{F}, \mathbf{I})$ is with $\frac{4}{15} \mathrm{nS} \leq g_{\text {gap }} \leq 4 \mathrm{nS}$. In each column, the transient generally last longest for homogeneous peripheral cell tissue (top row), following by homogeneous center cells, with the heterogeneous tissue having the shortest transient. The label $\mathbf{G}$ is red to correspond with the top red oval in Figure $6 \mathrm{E}$. 
except for very small $g_{g a p}$ values. The reduced model predicts that for very weak coupling, periodic solutions do not exist (blue), which we investigated further below (see Fig 11 A,B,C). Interestingly, there is a region where the peripheral-to-center traveling waves exist but have such large frequencies that the subsequent waves initiate before the previous one finishes (yellow in $\mathrm{Fig} 10 \mathrm{~B}$ ), resulting in phase lags that are non-monotonic; we investigated this response further in Fig $11 \mathrm{D}, \mathbf{E}, \mathbf{F}$. The tissue frequency when periodic solutions exist (Fig $10 \mathbf{D}, \mathbf{E}, \mathbf{F}$, same color scale) show no dependence on initial condition and decrease with coupling strength (consistent with Fig 5). These results validate our assertion that the traveling waves are locally stable (Fig 4 S1) for many initial conditions. The right column (Fig $10 \mathrm{G}, \mathbf{H}, \mathbf{I}$, same color scale) shows how the transient times depend on initial condition and coupling strength (lower range of $g_{g a p}$ shown because times are minuscule with larger $g_{\text {gap }}$ ). Transient times decrease with coupling strength and also decrease as the initial conditions are 'closer' to peripheral waves (bottom of $y$-axis), which is the stable periodic solution (except for blue and yellow in B). Thus, we have used the reduced phase model to both verify key aspects of the spatio-temporal dynamics over a large region of parameter space and identify regimes of irregular behavior, investigated further below.

From the large-scale tissue simulations, we identified a single parameter set for which a traveling wave solution likely did not exist, a heterogeneous tissue with stronger peripheral gap junction coupling. The reduced model further predicted a regime of parameter conditions for which traveling wave solutions likely do not exist and for which the single parameter set falls within, $\frac{0.5}{15} \mathrm{nS} \leq g_{\text {gap }} \leq 0.5 \mathrm{nS}$ (see Fig. 10 B in blue). The large-scale tissue simulation with heterogeneous tissue and stronger peripheral gap junction coupling thus validates the reduced model prediction of irregular dynamics. In this tissue, we find that the power spectrum of all 271 voltage traces (after transients have decayed) have a subset of 37 cells with multiple peaks (Fig. 11/A). This suggests that the voltage time course for some cells have multiple time-scales, in contrast to previously described tissues thus far, for which the voltage trace power spectrum is unimodal for all cells. Inspection of the voltage traces indeed shows irregularities from cycle to cycle (Fig. 11B), with different spike widths, heights, etc. for the subset of cells with multimodal power spectrums. The voltage trajectories of these 'irregular' cells are all synchronized except for the center most cell (see Fig. $11 \mathrm{C}$ for the spatial location). Importantly, the failure to find a traveling wave solution in the reduced model is consistent with the large-scale simulations, where the persistent solution is indeed not a traveling wave solution, demonstrating that the reduced model formulation can provide an approach to identify regimes of irregular voltage dynamics.

Recall the yellow region in Figure $10 \mathrm{~B}$ (strong peripheral coupling) where the heterogeneous phase model predicts a very fast peripheral-to-center traveling wave, such that the phase lags are non-monotonic (Fig.11D). We verified this prediction with corresponding large-scale simulations for $\frac{0.6}{15} \mathrm{nS} \leq g_{\text {gap }} \leq 0.6 \mathrm{nS}$. The power spectrum of the voltage (after transients have decayed) for all 271 cells have a single peak ( Fig $11 \mathrm{E}$ ), verifying the existence of a periodic solution. Finally, Figure 11F shows snapshots of the voltage (scaled by max and minimum voltage to compare across different cells) that demonstrate peripheral-to-center traveling waves, with dynamics such that the next wave initiates before the previous one ends, as predicted by the reduced phase model.

\subsection{Effects of Varying Gap Junction Coupling}

Above we described how the precise distribution of gap junction coupling strengths is still unknown despite ultrastructure studies because of the irregular structure and other components 



Frequency of Periodic Solution

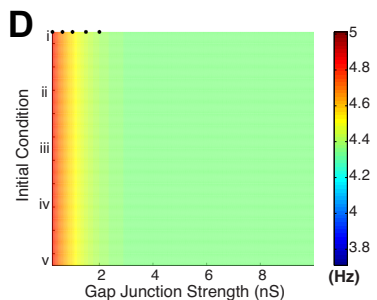

E

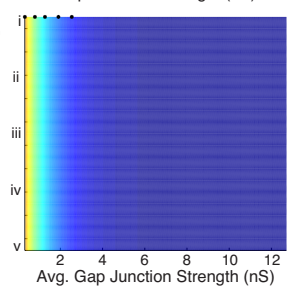

$\mathbf{F}$

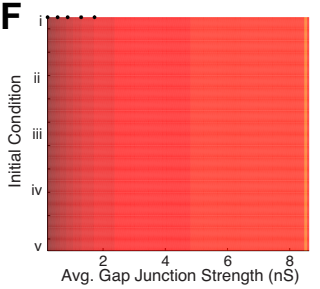

Transient Time

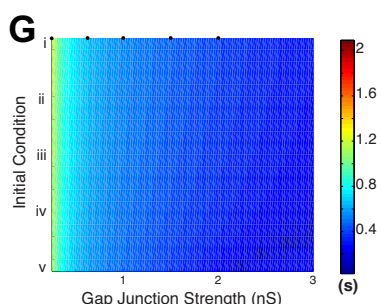

Gap Junction Strength (nS)

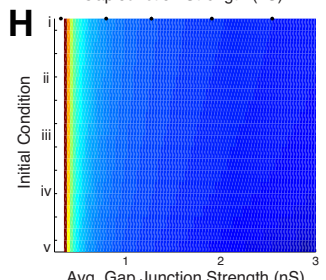

Avg. Gap Junction Strength (nS)

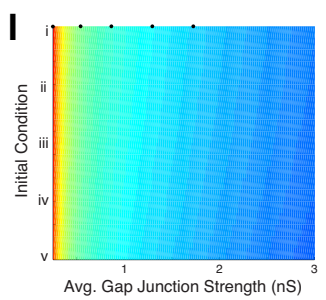

Figure 10: Phase model results with many initial conditions and coupling strengths for heterogeneous tissues. Top panel (gray): illustrative subset of the 21 initial conditions we consider, gradually varying from being primed for centerto-peripheral traveling waves (i), to starting at synchrony (iii), to being primed for peripheral-to-center waves (v). The large-scale simulations we previously considered correspond to the five black stars (A-I). With constant gap junction coupling (A) and with stronger central gap junction $(\mathbf{C})$, pertipheral-to-center traveling wave solutions exist (green) for all $g_{\text {gap }}$ sets and initial conditions considered. B) With stronger peripheral coupling, peripheral-to-center traveling waves exist for almost all parameters, the exception is with smaller coupling values where periodic solutions either do not exist (blue) or have very fast frequencies with non-monotnic phase lags (yellow, see Fig 11D,E,F for further details). The tissue frequency when periodic solutions exist for all 3 coupling schemes (D,E,F), same color scale) show no dependence on initial condition and decrease as coupling strength increases (cf. Fig 5]. The transient times (G,H,I, same color scale) decrease as coupling strength increases, and also decrease as initial conditions vary from (i) to (v) because the system starts 'closer' to peripheral-to-center traveling waves (i.e., the stable periodic solution). 



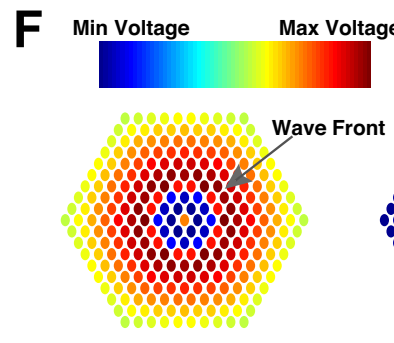

Time $=9.88 \mathrm{~s}$
- Multiple Peaks in Center cell $\longrightarrow$ Peripheral cell

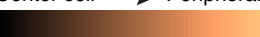

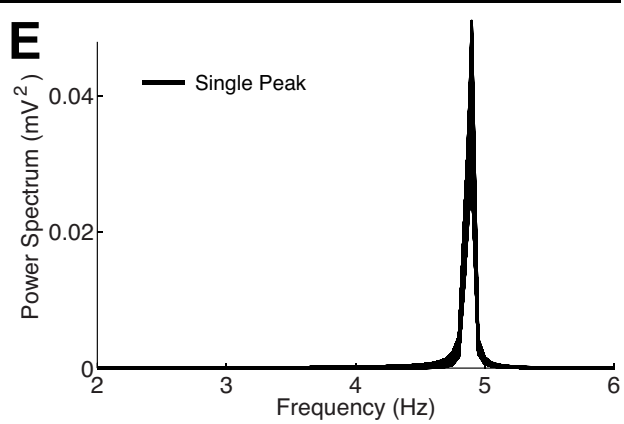

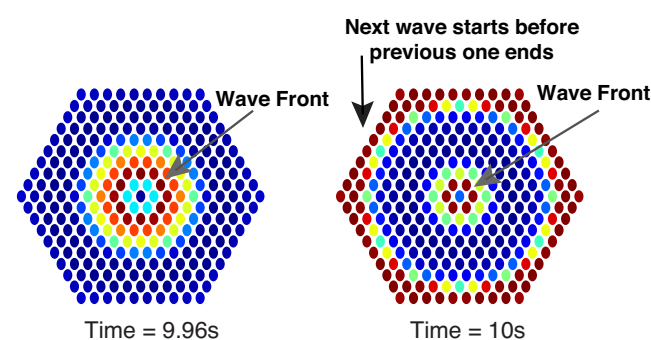

Figure 11: Irregular behavior in phase models correspond to irregular behavior in the large-scale models. A tissue structure and coupling scheme, for which a traveling wave solution does not exist A-C. The heterogeneous tissue has stronger peripheral gap junction coupling, with $\frac{0.5}{15} \mathrm{nS} \leq g_{\text {gap }} \leq 0.5 \mathrm{nS}$. A) Power spectrums of all 271 voltage traces (after transients have decayed, from $10 \mathrm{~s}-20 \mathrm{~s}$ ). There are cells (37 total in blue and magenta) with multiple peaks in the power spectrum, a signature of multiple time-scales; in contrast to other parameters considered. B) Two types of voltage traces that have irregular dynamics (different spike widths, heights, etc. from cycle to cycle); all but the center most cell (magenta) have the blue voltage traces. C) The spatial location of the cells with multiple time-scales in voltage. See Supplementary Material (vidx_Het_g2_s1.mp4 in S2.zip). D)-F) Again with strong peripheral gap junction coupling but with $\frac{0.6}{15} \mathrm{nS} \leq g_{\text {gap }} \leq 0.6 \mathrm{nS}$, corresponding to the yelfow region in Fig $10 \mathbf{B}$. The phase model predicts peripheralto-center waves that are so fast that the phase lags are non-monotonic $(\mathbf{D})$. E) The power spectrum of all cells in the corresponding large-scale simulation has 1 peak, like all other tissues with traveling waves. F) Snapshots of the largescale model in time show that the phase model prediction is verified, show peripheral-to-center traveling waves are so fast that the next wave starts before the prior one ends. See Supplementary Material (vidx_Het_gSMp6_s1.mp4 in S2.zip). 
in SAN, which motivated us to consider the three different coupling schemes. Thus far we have chosen modest gap junction values (up to $4 \mathrm{nS}$ for some pairs) in the large-scale simulations that have enabled analysis of how coupling values alter existence of traveling waves, tissue frequency, transient times, and have enabled comparisons of these entities across tissue models with different cell types. Experiments by Verheule et al. (2001) in rabbit SAN report an average $g_{g a p}$ value of $7.5 \mathrm{nS}$, with the median value among their 32 pairs of $5.3 \mathrm{nS}$ and a range from 0.6 to $25 \mathrm{nS}$. However, these measurements are performed in intact cell pairs and does not account for effectively reduced coupling between cells in the setting of highly fibrous SAN tissue. Reduced coupling can be further exacerbated with age and SAN disease (Jones et al., 2004). Therefore, we perform additional large-scale simulations over a wide range of coupling strengths; for constant gap junction coupling we set $g_{\text {gap }}=5,7.5,10 \mathrm{nS}$, for both types of gradient coupling (stronger central or stronger peripheral) the values varied from $\frac{10}{15} \mathrm{nS} \leq g_{\text {gap }} \leq 10 \mathrm{nS} ; 1 \mathrm{nS} \leq g_{\text {gap }} \leq 15 \mathrm{nS} ; \frac{20}{15} \mathrm{nS} \leq g_{\text {gap }} \leq 20 \mathrm{nS}$.

Simulations are compared with the corresponding reduced phase model (see Fig. S6) as in Figures 5 and 6 even though the approximations rely on weak coupling, and our prior results still qualitatively hold (i.e., reduced model qualitatively and sometimes quantitatively captures the trend of tissue frequency). The tissue frequencies for the heterogeneous tissue saturate with larger coupling values (Fig. S6A-C), while the transient times are very small for all tissue types (Fig. S6D-F). The detailed voltage trajectories for these larger $g_{g a p}$ values are shown in Figs. S7S9. Other large coupling values are shown here for completeness despite violating assumptions in the reduced phase model framework, but our main focus is on small to moderate coupling values that show more nuanced dynamics and entities (voltage, tissue frequency) consistent with experiments.

\section{Discussion}

In this study, we demonstrate the utility of the mathematical framework of coupled phase oscillators to mechanistically characterize the spatio-temporal dynamics of a large locally coupled tissue of heterogeneous sinoatrial node (SAN) cells, for which the individual cell models themselves are complex with many state variables and compartments. Summarizing our approach and main findings, we implemented a recently developed model of the central pacemaker cells in the SAN (Severi et al. 2012) and modified model parameters to capture a heterogeneous population of pacemaker cells to yield a large-scale SAN tissue tissue model. In electrically (gap junction) coupled tissue consisting of hundreds of pacemaker cells, we observed vastly different 'steadystate' or persistent behavior for tissue comprised of different intrinsic cell types. In these models, we found that the persistent behavior (synchrony for homogeneous cell tissue or peripheral-tocenter waves for heterogeneous tissue) was highly robust, even as both coupling schemes and gap junction strengths varied.

To explain observations in the large-scale models, we applied a reduced model framework consisting of a 1D chain of phase oscillators that accounted for different cell types and coupling strengths from the full 2D tissue. Importantly, the reduced phase model relied on quantities (i.e., intrinsic frequencies, cell-to-cell interactions) from the individual pacemaker cell model but did not rely on computationally expensive simulations of the large-scale tissue model.

The key novel results and insights of our study can be summarized as follows: 1) The reduced phase model framework successfully captured when traveling waves exist, uncovered the stability of these persistent states, and was used to calculate the transient times - all in a variety 
of tissue types, and coupling schemes and strengths. 2) In addition to requiring less computational resources to capture the large-scale tissue phenomena, the phase models facilitate detailed mathematical analysis and wider parameter studies of initial conditions and coupling schemes. 3) Despite incorporating a highly detailed biophysical model of SAN dynamics at the individual cell level, the large-scale tissue simulations predict that center-to-peripheral wave generation is only a transient behavior. Additionally, a wide parameter study in the reduced phase model further predicts that this finding holds, with the duration of transients depending on coupling strength and initial conditions. This demonstrates that additional model components are necessary to reproduce the physiologically observed center-to-peripheral wave generation. Note that experiments on rabbit SAN where the atrium is removed show peripheral-to-center traveling waves (Kirchhof et al. 1987), consistent with our heterogeneous model (also see Kodama and Boyett (1985); Opthof et al. (1987) for experiments that further validate our peripheral traveling wave results). Our future work will focus on addressing this model limitation, considering two potential mechanisms: i) incorporating additional anatomical details, specifically the electrical coupling between the SAN and the atria, and ii) incorporating spatially variable sensitivity to autonomic regulation that regulates pacemaker intrinsic frequency.

Phase reduction methods to explore dynamics of SAN models has a long history, albeit with less physiological models than the Severi et al. (2012) models we consider here. Many of the works we review here exploit simple models using a phase-resetting/response curve (PRC) or equivalent entity that quantifies how weak perturbations alter the time to the next action potential. The phase models we employ in this paper depend on the PRC, $\Delta$, but the method of averaging is used to yield interaction functions $H$ (see $\Delta$ in Eq.44). Ikeda (1982) and colleagues used minimal models with PRC functions based on experimental recordings of myocardial pacemaker cells in mammals (rabbit, cat) to mathematically analyze how pairs of cells can result in the existence of synchronized solutions - with simulations, they also considered 1D chains and 2D 'sheets' of pacemaker cells. Ikeda et al. (1986) and de Bruin et al. (1983) used simulations to probe how altering PRCs shape rhythms and synchrony. Based on simulations, AbramovichSivan and Akselrod studied the spatiotemporal patterns of a minimal SAN model (AbramovichSivan and Akselrod, 2000) with a PRC similar in form to Ikeda and colleagues; they also studied how the location and duration of traveling waves change with different intrinsic cycle lengths and PRCs (Abramovich-Sivan and Akselrod, 1999b), and how the atrium alters activity patterns (Abramovich-Sivan and Akselrod, 1999a). Östborn et al. (2001) employed a circle map simplification (i.e., no differential equations) of simple SAN models to determine entrainment dynamics and different locking states (i.e., n:m locking, beyond traveling waves). Östborn and colleagues studied how coupling strengths can lead to entrainment of cells with different cycle lengths (Östborn, 2002, 2004), rather than how various PRC shapes alter entrainment.

A number of researchers have considered models with different intrinsic cycle lengths (AbramovichSivan and Akselrod, 1999b a, 2000; Ikeda, 1982; Ikeda et al., 1986, de Bruin et al., 1983, Östborn et al. 2001; Östborn, 2002, 2004, Mirollo and Strogatz, 1990, Acebrón et al. | 2005, Mazurov 2006). These studies have typically used a 'pulse-coupling' approximation (effects of coupling only occur at one time point of the action potential) to model gap junction coupling, whereas our analysis does not require this. The main advantage of 'pulse-coupling' is apparently to have a well-defined set of coupled maps that (perhaps) are easier to analyze, rather than having a system of differential equations where the effects of coupling persists even when cells are not spiking. Another difference is that these studies (Abramovich-Sivan and Akselrod, 1999b a 2000. Östborn et al. 2001, Östborn, 2002, 2004; Ikeda, 1982, Ikeda et al. 1986, de Bruin et al., 1983) assume the PRCs can be manipulated in any desired way, whereas PRCs (and interaction func- 
tions) computed from biophysically detailed models have a complex dependence on parameters - they cannot be precisely controlled. Note that there may be other topological properties of the PRC when accounting for cell type that could be explored in the future (Guevara and Jongsma 1990, Ikeda et al., 1986; de Bruin et al., 1983). In contrast to these referenced works, we have focused on quantitative accuracy of the phase-reduced models with the large-scale models, as well as the shortcomings of the reduced phase models.

While the general analysis we used here has been applied to minimal cardiac cell models (i.e., see section 14.2 of Keener and Sneyd (1998)), to our knowledge, our analysis of a complex and highly detailed biophysical representation of pacemaker cells is unique and novel. Thus, our application is valuable because it establishes a framework for future mechanistic investigations of irregular SAN electrical function, driven by defective ion channel gating or expression. Ion channel dysfunction (e.g., reduced funny current associated with sick sinus syndrome (Schweizer et al. 2014)) can be incorporated into the underlying pacemaker cell models and subsequent reduced phase model stability can be predicted. As discussed in the previous paragraph, the application of reduced phase models with direct comparisons to large-scale heterogeneous tissue has been scarce; see (Ly, 2014, Zhou et al., 2013, Ashwin et al., 2016) for some exceptions. Regarding heterogeneity with the SAN tissue model, in terms of both cell type and cell-cell coupling gradients, we note that the large-scale model of 271 cells represents a subset of the full SAN tissue, and therefore a limitation of our model is that the steepness of parameter gradients is larger than that of a full-sized tissue. However, we chose to consider the full range of parameter conditions present in the full-size tissue (i.e., center-to-peripheral cell gradients, and cell-cell coupling gradients), a trade-off at the expense of a steeper gradient.

Our work could potentially elucidate large simulation studies in which center-to-peripheral traveling waves are observed. We do observe central-to-peripheral traveling waves, but only transiently, and the transient times strongly depend on cell types and coupling within the tissue. Specifically, the transients times were generally longer for homogeneous tissue than heterogeneous. In principle, this framework could be applied to other models (Zhang et al., 2000, Oren and Clancy, 2010) to mathematically characterize the steady-states and transient times. We note that Oren and Clancy (2010) argued that more realistic center-to-peripheral traveling waves only occurred in homogeneous tissue, and not in heterogeneous tissue (Joyner and Van Capelle (1986) also showed this in a homogeneous network). Other modelers have shown that centerto-peripheral waves with heterogeneous cells when SAN is coupled to the atrium: Inada et al. (2014) showed this with the Kurata model, see Unudurthi et al. (2014) for a review.

We have seen that there are regimes for which traveling wave solutions do not exist in the phase model (nor in the large-scale model) and the cell behavior is irregular from cycle to cycle (Fig. 11). Extending the phase oscillator framework to these complex dynamics and beyond is a promising area of future research, especially given the implications for these dynamics on cardiac arrhythmias and other disease. We are also interested in developing reduced phase model methods to analyze cardiac SAN tissue models that incorporate additional physiological features: further SAN cell population heterogeneity, including spatial heterogeneity in sensitivity to autonomic regulation and shifts in pacemaker origin (Csepe et al., 2016); incorporating both regions of electrical isolation and preferential conduction pathways between the SAN and the atria that have been identified in human and canine SAN (Fedorov et al., 2012); and including coupling with fibroblasts. Consistent with prior computational studies (Garny et al. 2003), we expect that the hyperpolarizing influence of the atrium would reduce the frequency of the intrinsically faster peripheral cells. Developing the mathematical analysis for a phase model with coupling between oscillatory pacemaker cells, excitable atrial cells, and non-excitable fibrous tissue is also a novel 
area for future work. Although phase oscillator theory is well-developed (Ashwin et al., 2016), and there is some theoretical progress in defining a phase of an excitable cells (Thomas and Lindner. 2014; Schwabedal and Pikovsky, 2013), a unified theory to succinctly describe oscillators coupled to excitable tissue is lacking. We hypothesize that these additional model details may yield stable center- to-peripheral traveling waves and also shifting origins of pacemaker rhythms in response to autonomic activity.

As experimental techniques advance and single cell models naturally increase in complexity with more variables and parameters, the necessity of tractable and insightful models will be dire. We have demonstrated that these reduced phase models and corresponding mathematical analysis are valuable for studying large realistic coupled cardiac tissues. For example, as more accurate data become available about the distribution of pacemaker cells' intrinsic frequency and cell coupling, this framework can be developed and extended to probe the spatio-temporal dynamics efficiently rather than performing computationally intensive simulations for a wide range of parameters.

\section{Appendix A. Detailed equations for the large-scale model}

Here we provide more details for the full pacemaker cell models (Eq. (1)). For the complete details, we provide the Matlab code for the models, publicly available at http://github.com/chengly70/SanHeteroSeveri. The complete details of the center cell pacemaker model is provided in Severi et al. (2012), with computer code publicly available at http://models.cellml.org/e/139/severi_fantini_charawi_difrancesco_2012.cellml/view. There are 31 total dynamic variables with their own differential equation for each cell, and many auxiliary functions.

The voltages are in units of milli-Volts $(\mathrm{mV})$. The sodium current is:

$$
\begin{aligned}
I_{N a}(v) & =g_{N a} m^{3} h\left(v-E_{N a}\right) \\
E_{N a} & =\frac{R T}{F} \ln \left(N a_{o} / N a_{i}\right) \\
\frac{d x}{d t} & =\alpha_{x}(v)(1-x)-\beta_{x}(v) x, \text { where } x \in\{m, h\}
\end{aligned}
$$

where $g_{N a}$ is the conductance (varies, see Table 2), $m$ and $h$ are the sodium activation and inactivation variables, $R$ is the universal gas constant, $F$ is Faraday's constant, $T$ is temperature in Kelvin $\left(310\right.$, or $\left.36.85^{\circ} \mathrm{C}\right), N a_{o}=140 \mathrm{mM}$ is the extracellular sodium concentration; $N a_{i}$ is the dynamic intracellular concentration with its own differential equation; $\alpha_{m}(v), \alpha_{h}(v), \beta_{m}(v), \beta_{h}(v)$ are standard gating variables.

The funny current is:

$$
\begin{aligned}
I_{f}(v) & =g_{f, N a} y_{f}^{2} K_{o} /\left(K_{o}+45\right)\left(v-E_{N a}\right)+g_{f, K} y_{f}^{2} K_{o} /\left(K_{o}+45\right)\left(v-E_{K}\right) \\
E_{K} & =\frac{R T}{F} \ln \left(K_{o} / K_{i}\right) \approx-86.96 \mathrm{mV} \\
\frac{d\left(y_{f}\right)}{d t} & =\left(y_{f, \infty}(v)-y_{f}\right) / \tau_{y f}(v)
\end{aligned}
$$

where $g_{f, N a}, g_{f, K}$ are the conductances (varies, see Table 2); $K_{o}=5.4 \mathrm{mM}$ is the extracellular potassium concentration, $K_{i}=140 \mathrm{mM}$ is the intracellular potassium concentration. 
The transient outward potassium current is:

$$
\begin{aligned}
I_{t o}(v) & =g_{t o} q * r *\left(v-E_{K}\right) \\
\frac{d x}{d t} & =\left(x_{\infty}(v)-x\right) / \tau_{x}(v), \text { where } x \in\{q, r\}
\end{aligned}
$$

with $g_{t o}=0.002 \mu \mathrm{S}$; for this and other currents, the steady state functions $x_{\infty}(v)$ and time constant $\tau_{x}(v)$ are standard (see Severi et al. (2012)).

The L-type calcium current is:

$$
\begin{aligned}
& I_{\mathrm{Ca}, \mathrm{L}}=I_{s i, \mathrm{Ca}}+I_{s i, \mathrm{~K}}+I_{s i, \mathrm{Na}} \\
& I_{s i, C a}=\frac{2 P_{C a L} * v}{\frac{R T}{F}\left(1-\exp \left(-2 v \frac{F}{R T}\right)\right)}\left(C a_{s u b}-C a_{o} \exp \left(-2 v \frac{F}{R T}\right)\right) * d_{L} * f_{L} * f_{C a} \\
& I_{s i, K}=\frac{3.65 \times 10^{-4} P_{C a L} * v}{\frac{R T}{F}\left(1-\exp \left(-v \frac{F}{R T}\right)\right)}\left(K_{i}-K_{o} \exp \left(-v \frac{F}{R T}\right)\right) * d_{L} * f_{L} * f_{C a} \\
& I_{s i, N a}=\frac{1.85 \times 10^{-5} P_{C a L} * v}{\frac{R T}{F}\left(1-\exp \left(-v \frac{F}{R T}\right)\right)}\left(N a_{i}-N a_{o} \exp \left(-v \frac{F}{R T}\right)\right) * d_{L} * f_{L} * f_{C a} \\
& \frac{d x}{d t}=\left(x_{\infty}(v)-x\right) / \tau_{x}(v), \text { where } x \in\left\{d_{L}, f_{L}\right\} \\
& \frac{d\left(f_{C a}\right)}{d t}=\left(f_{C a, \infty}\left(C a_{s u b}\right)-f_{C a}\right) / \tau_{f C a}\left(C a_{s u b}\right)
\end{aligned}
$$

where $P_{C a L}$ is the conductance (varies, see Table 2), $C a_{o}=1.8 \mathrm{mM}$ is the extracellular calcium concentration, $C a_{\text {sub }}$ is the dynamic subspace calcium concentration with its own differential equation.

The T-type calcium current is:

$$
\begin{aligned}
I_{C a, T} & =\frac{2 P_{C a T} * v}{\frac{R T}{F}\left(1-\exp \left(-2 v \frac{F}{R T}\right)\right)}\left(C a_{s u b}-C a_{o} \exp \left(-2 v \frac{F}{R T}\right)\right) * d_{T} * f_{T} \\
\frac{d x}{d t} & =\left(x_{\infty}(v)-x\right) / \tau_{x}(v), \text { where } x \in\left\{d_{T}, f_{T}\right\}
\end{aligned}
$$

where $P_{C a T}$ is the conductance (varies, see Table 2).

The rapid delayed rectifier $\mathrm{K}^{+}$and slow delayed rectifier $\mathrm{K}^{+}$currents are:

$$
\begin{aligned}
I_{K r} & =g_{K r} *\left(0.9 * p_{a F}+0.1 * p_{a S}\right) * p_{i} *\left(v-E_{K}\right) \\
I_{K s} & =g_{K s} * n^{2} *\left(v-E_{K}\right) \\
\frac{d x}{d t} & =\left(x_{\infty}(v)-x\right) / \tau_{x}(v), \text { where } x \in\left\{p_{a F}, p_{a S}, p_{i}, n\right\}
\end{aligned}
$$

where both $g_{K r}$ and $g_{K s}$ conductances vary by cell type (see Table 2).

The sodium-potassium pump current $I_{\mathrm{Na}, \mathrm{K}}$ and sodium-calcium exchanger current $I_{\mathrm{Na}, \mathrm{Ca}}$ are:

$$
\begin{aligned}
I_{N a, K} & =\frac{g_{N a K}}{\left(1+1.4 / K_{o}\right)^{1.2}\left(1+14 / N a_{i}\right)^{1.3}\left(1+\exp \left(-\left(v-E_{N a}+110\right) / 20\right)\right)} \\
I_{N a, C a} & =g_{N a C a} \frac{x_{2} k_{21}-x_{1} k_{12}}{x_{1}+x_{2}+x_{3}+x_{4}}
\end{aligned}
$$


The components $x_{1}, \ldots, x_{4}$ and $k_{21}, k_{12}$ represent the detailed calcium dynamics in the sarcoplasmic recticulum (SR), myoplasm, junctional SR, subspace SR, as well as outside of the cell. We omit the rest of the equations and functions due to their complexity and point the interested reader to our freely available computer code. We alter cell length and cell radius for different cell types, that in turns effects the cell volume, submembrane space volume, myoplasmic volume, junctional SR volume, network SR volume, which all effect the calcium dynamics.

The values for $g_{\mathrm{NaK}}$ and $g_{\mathrm{NaCa}}$ for the 9 different cells were manually altered to insure ion homeostasis and a stable limit cycle in the uncoupled cells, the values are listed in Table 3.

\section{Acknowledgements}

The authors acknowledge and thank the Virginia Commonwealth University Center for High Performance Computing (CHiPC) for use of the Teal cluster to perform numerical simulations. CL is supported by a grant from the Simons Foundation (\# 355173).

\section{Supplementary Material}

SlText.pdf-Supplementary Figures

A PDF files that contains 9 extra figures that expound on Figures 4, 6B,D,F, 7,9, and show the results for large gap junction strengths.

S2.zip-Movies

A zip file containing several movie files (.mp4) showing the voltage evolution on the $2 \mathrm{D}$ hexagonal grid. Naming convention:

vidx_[Het/HomC/HomP]_io_g [2/22/3/33/4]_s [1/2/3] .mp4

Het is with all cell types (heterogeneous), HomC is a homogeneous tissue with all center cells, HomP is a homogeneous tissue with all peripheral cells.

s1: gradient gap strength with peripheral having $15 \mathrm{X}$ larger strength than center.

s2: gradient gap strength with center having $15 \mathrm{X}$ larger strength than peripheral.

s3: constant gap strength throughout.

g2 $=0.25 \mathrm{nS}\left([0.5 / 15,0.5] \mathrm{nS}\right.$ range of $g_{\text {gap }}$ when gradient gap strength s1 \& s2)

g22 $=0.625 \mathrm{nS}([1.25 / 15,1.25] \mathrm{nS}$ range with gradient gap schemes)

$\mathrm{g} 3=1 \mathrm{nS}([2 / 15,2] \mathrm{nS}$ range with gradient gap schemes)

$\mathrm{g} 33=1.5 \mathrm{nS}([3 / 15,3] \mathrm{nS}$ range with gradient gap schemes)

$\mathrm{g} 4=2 \mathrm{nS}([4 / 15,4] \mathrm{nS}$ range with gradient gap schemes)

Also two movie files, vidx_Het_gSMp $[6,7] \_s 1 . m p 4$, correspond to 2 points in the yellow region of Figure $10 \mathbf{B}$ with initial condition (i).

\section{References}

\section{References}

Abramovich-Sivan, S., Akselrod, S., 1999a. Phase response curve based model of the sa node: simulation by twodimensional array of pacemaker cells with randomly distributed cycle lengths. Medical \& biological engineering \& computing 37 (4), 482-491.

Abramovich-Sivan, S., Akselrod, S., 1999b. Simulation of atrial activity by a phase response curve based model of a two-dimensional pacemaker cells array: the transition from a normal activation pattern to atrial fibrillation. Biological cybernetics 80 (2), 141-153. 
Abramovich-Sivan, S., Akselrod, S., 2000. A simulation of the sa node by a phase response curve-based model of a two-dimensional pacemaker cells array. IEEE transactions on biomedical engineering 47 (4), 425-434.

Acebrón, J. A., Bonilla, L. L., Vicente, C. J. P., Ritort, F., Spigler, R., 2005. The kuramoto model: A simple paradigm for synchronization phenomena. Reviews of modern physics 77 (1), 137.

Arevalo, H. J., Boyle, P. M., Trayanova, N. A., 2016. Computational rabbit models to investigate the initiation, perpetuation, and termination of ventricular arrhythmia. Progress in biophysics and molecular biology 121 (2), 185-194.

Ashwin, P., Coombes, S., Nicks, R., 2016. Mathematical frameworks for oscillatory network dynamics in neuroscience The Journal of Mathematical Neuroscience 6 (1), 2.

Bleeker, W. K., Mackaay, A. J., Masson-Pévet, M., Bouman, L. N., Becker, A. E., 1980. Functional and morphologica organization of the rabbit sinus node. Circulation research 46 (1), 11-22.

Bouman, L., Jongsma, H., 1986. Structure and function of the sino-atrial node: a review. European heart journal 7 (2), 94-104.

Boyett, M. R., Honjo, H., Kodama, I., 2000. The sinoatrial node, a heterogeneous pacemaker structure. Cardiovascular research 47 (4), 658-687.

Csepe, T. A., Zhao, J., Hansen, B. J., Li, N., Sul, L. V., Lim, P., Wang, Y., Simonetti, O. P., Kilic, A., Mohler, P. J., et al., 2016. Human sinoatrial node structure: $3 \mathrm{~d}$ microanatomy of sinoatrial conduction pathways. Progress in biophysics and molecular biology 120 (1), 164-178.

de Bruin, G., Ypey, D. L., Van Meerwijk, W. P., 1983. Synchronization in chains of pacemaker cells by phase resetting action potential effects. Biological cybernetics 48 (3), 175-186.

Efimov, I. R., Fedorov, V. V., Joung, B., Lin, S.-F., 2010. Mapping cardiac pacemaker circuits. Circulation research 106 (2), 255-271

Ermentrout, B., 2002. Simulating, analyzing, and animating dynamical systems: a guide to XPPAUT for researchers and students. SIAM.

Ermentrout, G. B., 1992. Stable periodic solutions to discrete and continuum arrays of weakly coupled nonlinear oscillators. SIAM Journal on Applied Mathematics 52 (6), 1665-1687.

Ermentrout, G. B., Terman, D. H., 2010. Mathematical foundations of neuroscience. Vol. 35. Springer Science \& Business Media.

Fedorov, V. V., Chang, R., Glukhov, A. V., Kostecki, G., Janks, D., Schuessler, R. B., Efimov, I. R., 2010. Complex interactions between the sinoatrial node and atrium during reentrant arrhythmias in the canine heart. Circulation $122(8), 782-789$

Fedorov, V. V., Glukhov, A. V., Chang, R., 2012. Conduction barriers and pathways of the sinoatrial pacemaker complex: their role in normal rhythm and atrial arrhythmias. American Journal of Physiology-Heart and Circulatory Physiology 302 (9), H1773-H1783.

Fedorov, V. V., Schuessler, R. B., Hemphill, M., Ambrosi, C. M., Chang, R., Voloshina, A. S., Brown, K., Hucker, W. J., Efimov, I. R., 2009. Structural and functional evidence for discrete exit pathways that connect the canine sinoatrial node and atria. Circulation research 104 (7), 915-923.

Garny, A., Kohl, P., Hunter, P. J., Boyett, M. R., Noble, D., 2003. One-dimensional rabbit sinoatrial node models. Journal of cardiovascular electrophysiology 14 (s10).

Glukhov, A. V., Hage, L. T., Hansen, B. J., Pedraza-Toscano, A., Vargas-Pinto, P., Hamlin, R. L., Weiss, R., Carnes, C. A., Billman, G. E., Fedorov, V. V., 2013. Sinoatrial node reentry in a canine chronic left ventricular infarct model: The role of intranodal fibrosis and heterogeneity of refractoriness. Circulation: Arrhythmia and Electrophysiology, CIRCEP-113.

Greer-Short, A., George, S. A., Poelzing, S., Weinberg, S. H., 2017. Revealing the concealed nature of long-qt type 3 syndrome. Circulation: Arrhythmia and Electrophysiology 10 (2), e004400.

Guevara, M. R., Jongsma, H. J., 1990. Phase resetting in a model of sinoatrial nodal membrane: ionic and topological aspects. American Journal of Physiology-Heart and Circulatory Physiology 258 (3), H734-H747.

Honjo, H., Boyett, M., Kodama, I., Toyama, J., 1996. Correlation between electrical activity and the size of rabbit sino-atrial node cells. The Journal of Physiology 496 (3), 795-808

Ikeda, N., 1982. Model of bidirectional interaction between myocardial pacemakers based on the phase response curve. Biological cybernetics 43 (3), 157-167.

Ikeda, N., Yamamoto, H., Sato, T., 1986. Pathology of the pacemaker network. Mathematical Modelling 7 (5-8), 889904.

Inada, S., Zhang, H., Tellez, J. O., Shibata, N., Nakazawa, K., Kamiya, K., Kodama, I., Mitsui, K., Dobrzynski, H., Boyett, M. R., et al., 2014. Importance of gradients in membrane properties and electrical coupling in sinoatrial node pacing. PloS one 9 (4), e94565.

Jalife, J., Hamilton, A., Lamanna, V., Moe, G., 1980. Effects of current flow on pacemaker activity of the isolated kitten sinoatrial node. American Journal of Physiology-Heart and Circulatory Physiology 238 (3), H307-H316.

Jones, S. A., Lancaster, M. K., Boyett, M. R., 2004. Ageing-related changes of connexins and conduction within the sinoatrial node. The Journal of physiology 560 (2), 429-437. 
Joyner, R. W., Van Capelle, F., 1986. Propagation through electrically coupled cells. how a small sa node drives a large atrium. Biophysical Journal 50 (6), 1157-1164.

Keener, J., Sneyd, J., 1998. Mathematical physiology. Interdisciplinary applied mathematics, vol. 8. Springer-Verlag, New York.

Kirchhof, C. J., Bonke, F. I., Allessie, M. A., Lammers, W. J., 1987. The influence of the atrial myocardium on impulse formation in the rabbit sinus node. Pflügers Archiv 410 (1-2), 198-203.

Kodama, I., Boyett, M. R., 1985. Regional differences in the electrical activity of the rabbit sinus node. Pflügers Archiv 404 (3), 214-226.

Kodama, I., Nikmaram, M., Boyett, M., Suzuki, R., Honjo, H., Owen, J., 1997. Regional differences in the role of the ca2+ and na+ currents in pacemaker activity in the sinoatrial node. American Journal of Physiology-Heart and Circulatory Physiology 272 (6), H2793-H2806.

Kopell, N., Ermentrout, G., 1986. Symmetry and phaselocking in chains of weakly coupled oscillators. Communications on Pure and Applied Mathematics 39 (5), 623-660.

Kuramoto, Y., 2012. Chemical oscillations, waves, and turbulence. Vol. 19. Springer Science \& Business Media.

Kurata, Y., Hisatome, I., Imanishi, S., Shibamoto, T., 2002. Dynamical description of sinoatrial node pacemaking: improved mathematical model for primary pacemaker cell. American Journal of Physiology-Heart and Circulatory Physiology 283 (5), H2074-H2101.

Li, N., Csepe, T. A., Hansen, B. J., Dobrzynski, H., Higgins, R. S., Kilic, A., Mohler, P. J., Janssen, P. M., Rosen, M. R. Biesiadecki, B. J., et al., 2015. Molecular mapping of sinoatrial node hen channel expression in the human heart. Circulation: Arrhythmia and Electrophysiology, CIRCEP-115.

Li, N., Hansen, B. J., Csepe, T. A., Zhao, J., Ignozzi, A. J., Sul, L. V., Zakharkin, S. O., Kalyanasundaram, A., Davis, J. P., Biesiadecki, B. J., et al., 2017. Redundant and diverse intranodal pacemakers and conduction pathways protect the human sinoatrial node from failure. Science translational medicine 9 (400), eaam5607.

Ly, C., 2014. Dynamics of coupled noisy neural oscillators with heterogeneous phase resetting curves. SIAM Journal on Applied Dynamical Systems 13, 1733-1755.

Ly, C., Ermentrout, G. B., 2010. Analysis of recurrent networks of pulse-coupled noisy neural oscillators. SIAM Journal on Applied Dynamical Systems 9, 113-137.

Masson-Pévet, M., Bleeker, W. K., Gros, D., 1979. The plasma membrane of leading pacemaker cells in the rabbit sinus node. a qualitative and quantitative ultrastructural analysis. Circulation Research 45 (5), 621-629.

Mazurov, M., 2006. Rhythmogenesis in the sinoatrial unit of the heart. Biofizika 51 (6), 1092-1099.

Michaels, D. C., Matyas, E. P., Jalife, J., 1987. Mechanisms of sinoatrial pacemaker synchronization: a new hypothesis Circulation Research 61 (5), 704-714.

Mirollo, R. E., Strogatz, S. H., 1990. Synchronization of pulse-coupled biological oscillators. SIAM Journal on Applied Mathematics 50 (6), 1645-1662.

Opthof, T., VanGinneken, A. C., Bouman, L. N., Jongsma, H. J., 1987. The intrinsic cycle length in small pieces isolated from the rabbit sinoatrial node. Journal of molecular and cellular cardiology 19 (9), 923-934.

Oren, R. V., Clancy, C. E., 2010. Determinants of heterogeneity, excitation and conduction in the sinoatrial node: a model study. PLoS computational biology 6 (12), e1001041.

Östborn, P., 2002. Phase transition to frequency entrainment in a long chain of pulse-coupled oscillators. Physical Review E 66 (1), 016105

Östborn, P., 2004. Frequency entrainment in long chains of oscillators with random natural frequencies in the weak coupling limit. Physical Review E 70 (1), 016120

Östborn, P., Ohlén, G., Wohlfart, B., 2001. Simulated sinoatrial exit blocks explained by circle map analysis. Journal of theoretical biology 211 (3), 219-227.

Phadumdeo, V. M., Weinberg, S. H., 2018. Heart rate variability alters cardiac repolarization and electromechanical dynamics. Journal of theoretical biology.

Sano, T., Sawanobori, T., Adaniya, H., 1978. Mechanism of rhythm determination among pacemaker cells of the mammalian sinus node. American Journal of Physiology-Heart and Circulatory Physiology 235 (4), H379-H384.

Schultheiss, N. W., Prinz, A. A., Butera, R. J., 2011. Phase response curves in neuroscience: theory, experiment, and analysis. Springer Science \& Business Media.

Schwabedal, J. T., Pikovsky, A., 2013. Phase description of stochastic oscillations. Physical review letters 110 (20), 204102.

Schweizer, P. A., Schröter, J., Greiner, S., Haas, J., Yampolsky, P., Mereles, D., Buss, S. J., Seyler, C., Bruehl, C., Draguhn, A., et al., 2014. The symptom complex of familial sinus node dysfunction and myocardial noncompaction is associated with mutations in the hen4 channel. Journal of the American College of Cardiology 64 (8), 757-767.

Severi, S., Fantini, M., Charawi, L. A., DiFrancesco, D., 2012. An updated computational model of rabbit sinoatrial action potential to investigate the mechanisms of heart rate modulation. The Journal of physiology 590 (18), $4483-$ 4499

Thomas, P. J., Lindner, B., 2014. Asymptotic phase for stochastic oscillators. Physical review letters 113 (25), 254101. 
Unudurthi, S. D., Wolf, R. M., Hund, T. J., 2014. Role of sinoatrial node architecture in maintaining a balanced sourcesink relationship and synchronous cardiac pacemaking. Frontiers in physiology 5, 446.

Verheijck, E. E., Wessels, A., van Ginneken, A. C., Bourier, J., Markman, M. W., Vermeulen, J. L., de Bakker, J. M. Lamers, W. H., Opthof, T., Bouman, L. N., 1998. Distribution of atrial and nodal cells within the rabbit sinoatrial node: models of sinoatrial transition. Circulation 97 (16), 1623-1631.

Verheule, S., van Kempen, M. J., Postma, S., Rook, M. B., Jongsma, H. J., 2001. Gap junctions in the rabbit sinoatrial node. American Journal of Physiology-Heart and Circulatory Physiology 280 (5), H2103-H2115.

Wilders, R., 2007. Computer modelling of the sinoatrial node. Medical \& biological engineering \& computing 45 (2), 189-207.

Winfree, A. T., 1967. Biological rhythms and the behavior of populations of coupled oscillators. Journal of theoretical biology 16 (1), 15-42.

Winfree, A. T., 1970. Integrated view of resetting a circadian clock. Journal of Theoretical Biology 28 (3), $327-374$.

Zeitzer, J. M., Dijk, D.-J., Kronauer, R. E., Brown, E. N., Czeisler, C. A., 2000. Sensitivity of the human circadian pacemaker to nocturnal light: melatonin phase resetting and suppression. The Journal of physiology 526 (3), $695-$ 702.

Zhang, H., Holden, A., Kodama, I., Honjo, H., Lei, M., Varghese, T., Boyett, M., 2000. Mathematical models of action potentials in the periphery and center of the rabbit sinoatrial node. American Journal of Physiology-Heart and Circulatory Physiology 279 (1), H397-H421.

Zhou, P., Burton, S., Urban, N., Ermentrout, G. B., 2013. Impact of neuronal heterogeneity on correlated colored noiseinduced synchronization. Frontiers in computational neuroscience 7, 113. 


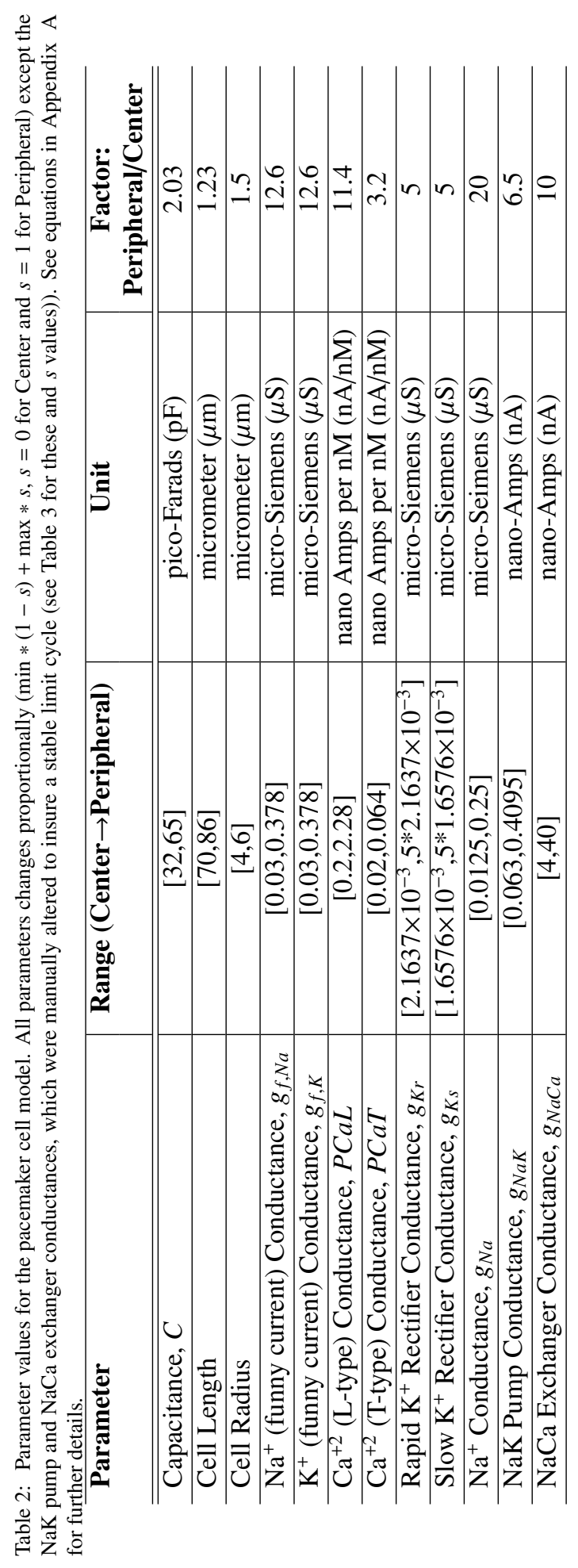


Table 3: Parameter values for $\mathrm{NaK}$ pump and $\mathrm{NaCa}$ exchanger current and $s$ proportionality scalar $\min *(1-s)+\max * s$.

\begin{tabular}{c|c|c|c}
\hline Cell Type & $g_{\text {NaK }}(\mathrm{nA})$ & $g_{\text {NaCa }}(\mathrm{nA})$ & $s \in[0,1]$ \\
\hline \hline 1 (center) & 0.063 & 4 & 0 \\
\hline 2 & 0.0695 & 6 & $0.25 / 9$ \\
\hline 3 & 0.08 & 6.7 & $0.65 / 9$ \\
\hline 4 & 0.095 & 8.9 & $1.2 / 9$ \\
\hline 5 & 0.125 & 13.5 & $2 / 9$ \\
\hline 6 & 0.164 & 17 & $3 / 9$ \\
\hline 7 & 0.2045 & 21 & $4 / 9$ \\
\hline 8 & 0.2871 & 29 & $6 / 9$ \\
\hline 9 (peripheral) & 0.4095 & 40 & 1 \\
\hline
\end{tabular}

